\title{
SIGN-CHANGING SOLUTIONS TO ELLIPTIC SECOND ORDER EQUATIONS: GLUEING A PEAK TO A DEGENERATE CRITICAL MANIFOLD
}

\author{
FRÉDÉRIC ROBERT AND JÉRÔME VÉTOIS
}

\begin{abstract}
We construct blowing-up sign-changing solutions to some nonlinear critical equations by glueing a standard bubble to a degenerate function. We develop a new method based on analyticity to perform the glueing when the critical manifold of solutions is degenerate and no Bianchi-Egnell type condition holds.
\end{abstract}

\section{InTRODUCTION AND STATEMENT OF THE RESUlts}

Let $(M, g)$ be a smooth compact Riemannian manifold of dimension $n \geq 3$, and let $h \in C^{0, \theta}(M)(\theta \in(0,1))$ be such that $\Delta_{g}+h$ is coercive where $\Delta_{g}=-\operatorname{div}_{g}(\nabla)$ is the Laplace-Beltrami operator. In 24], we addressed the question of the existence of a family $\left(u_{\varepsilon}\right)_{\varepsilon>0} \in C^{2, \theta}(M)$ of blowing-up solutions of type $\left(u_{0}-B\right)$ to

$$
\Delta_{g} u_{\varepsilon}+h u_{\varepsilon}=\left|u_{\varepsilon}\right|^{2^{\star}-2-\varepsilon} u_{\varepsilon} \text { in } M,
$$

where $2^{\star}:=\frac{2 n}{n-2}$. Concerning terminology, we say that $\left(u_{\varepsilon}\right)_{\varepsilon}$ is of type $\left(u_{0}-B\right)$ when there exists a function $u_{0} \in C^{2, \theta}(M)$ positive that is a solution to

$$
\Delta_{g} u_{0}+h u_{0}=u_{0}^{2^{\star}-1} \text { in } M
$$

and such that

$$
u_{\varepsilon}=u_{0}-B_{\varepsilon}+o(1)
$$

where $\left(B_{\varepsilon}\right)_{\varepsilon}$ is a bubble as defined in (6) below and $\lim _{\varepsilon \rightarrow 0} o(1)=0$ in $H_{1}^{2}(M)$, the completion of $C^{\infty}(M)$ for the norm $u \mapsto\|u\|_{H_{1}^{2}}:=\|u\|_{2}+\|\nabla u\|_{2}$. Solutions of type $\left(u_{0}-B\right)$ are sign-changing. When $h \equiv c_{n} R_{g}$, where $c_{n}:=\frac{n-2}{4(n-1)}$ and $R_{g}$ is the scalar curvature, equation (2) is the Yamabe equation, and $\Delta_{g}+h$ is coercive if and only if $(M, g)$ has positive Yamabe invariant. There is an extensive literature on the existence of positive blowing-up solutions to equations of type (1): see for instance Rey [23] for a historical reference, Brendle-Marques [4] for the Yamabe equation, Druet-Hebey [13] and Esposito-Pistoia-Vétois [14] for perturbations of the Yamabe equation, Chen-Wei-Yan [6] and Hebey-Wei [15] for equations on the sphere, and the references therein. Sign-changing blowing-up solutions to (1) on the canonical sphere have been constructed by del Pino-Musso-Pacard-Pistoia [10,11] and Pistoia-Vétois [22]. We refer to Robert-Vétois 24] for a discussion and references on the compactness of solutions to (11).

Date: September 8th, 2014.

The research of the first author is partially supported by INSMI (CNRS). 
In 24], we gave sufficient conditions to get blowing-up solutions of type $\left(u_{0}-B\right)$ to (11) provided that $u_{0}$ is a nondegenerate solution to (2), that is $K_{0}=\{0\}$ where

$$
K_{0}:=\left\{\varphi \in C^{2, \theta}(M) / \Delta_{g} \varphi+h \varphi=\left(2^{\star}-1\right) u_{0}^{2^{\star}-2} \varphi \text { in } M\right\} .
$$

When $u_{0}$ is degenerate, the situation can be different. In [24], we showed that there is no blowing-up solutions of type $\left(u_{0}-B\right)$ to the constant scalar curvature equation on the canonical sphere: in this case, $u_{0}$ is necessarily degenerate.

The present article is devoted to the analysis of the degenerate case, that is when $K_{0} \neq\{0\}$. We say that $u_{0} \in C^{2, \theta}(M) \backslash\{0\}$ is a strict local minimizer of $I_{0}$ if there exists $\nu>0$ such that

$$
I_{0}(u)>I_{0}\left(u_{0}\right) \text { for all } u \in B_{\nu}\left(u_{0}\right) \backslash \mathbb{R} u_{0},
$$

where

$$
I_{0}(u):=\frac{\int_{M}\left(|\nabla u|_{g}^{2}+h u^{2}\right) d v_{g}}{\left(\int_{M}|u|^{2^{\star}} d v_{g}\right)^{\frac{2}{2^{\star}}}}
$$

for all $u \in H_{1}^{2}(M) \backslash\{0\}$. Our main result is the following:

Theorem 1.1. We let $(M, g)$ be a compact Riemannian manifold of dimension $n \geq 3$ with positive Yamabe invariant and we fix $h \equiv \frac{n-2}{4(n-1)} R_{g}$. We assume that there exists $u_{0} \in C^{2, \theta}(M)$ that is a positive solution to (2) and a strict local minimizer of $I_{0}$. We assume either that $\{3 \leq n \leq 9\}$ or that $\{(M, g)$ is locally conformally flat $\}$. Then there exists a solution of type $\left(u_{0}-B\right)$ to (1).

It follows from the compactness results of Schoen [26] and Khuri-Marques-Schoen 17. (see also Druet [12) that blowing-up solutions to (1) must change sign under the assumptions of Theorem 1.1

As a remark, any nondegenerate local minimizer of $I_{0}$ is a strict local minimizer, so we recover the main theorem of [24]. Moreover no solution of the Yamabe equation on the sphere is a strict local minimizer. However, as soon as one takes the product of a sphere with another manifold, one gets examples of degenerate strict local minimizers. We refer to Section 7 for such examples, in particular to Corollary 7.1$]$

We prove Theorem 1.1 by performing a finite-dimensional reduction modeled on $(u-B)$ where $B$ is a bubble and $u \in \mathcal{M}$, and where $\mathcal{M}$ is a suitable finitedimensional analytic manifold containing $u_{0}$. We construct the manifold $\mathcal{M}$ such that its elements are as close as possible to solutions of (2): this is done using a first finite-dimensional reduction. The manifold $\mathcal{M}$ is locally parametrized by $K_{0}$, and the tangent space of $\mathcal{M}$ at $u_{0}$ is $K_{0}$. The general construction in Robert-Vétois [25] reduces the proof of Theorem 1.1 to finding stable critical points to a functional that is the sum of two terms: the first is an explicit local well involving essentially the bubble, the second is the restriction to $\mathcal{M}$ of a nontrivial global functional $J_{0}$.

Solutions to (2) around $u_{0}$ are all in $\mathcal{M}$. However, in general, the elements of $\mathcal{M}$ are not all solutions to (2), that is $\mathcal{M}$ is not a critical manifold of the problem. Following the terminology of Chapter 2 of the monograph Ambrosetti-Malchiodi 1], a critical manifold around $u_{0}$ is a finite-dimensional manifold $\mathcal{Z} \ni u_{0}$ of solutions to (2). A critical manifold $\mathcal{Z}$ is nondegenerate if its tangent space at any $u \in \mathcal{Z}$ is exactly $\operatorname{Ker}\left(I_{0}^{\prime \prime}(u)\right)$, the kernel of the Hessian of $I_{0}$ at $u$. The existence of a 
nondegenerate critical manifold around $u_{0}$ is equivalent to the existence of $\tilde{u} \in$ $C^{1}\left(B_{1}(0) \subset K_{0}, H_{1}^{2}(M)\right)$ such that

$$
\left\{\begin{array}{l}
\tilde{u}(z) \text { is a solution to (2) for all } z \in B_{1}(0) \subset K_{0}, \\
\tilde{u}(0)=u_{0}, \\
K_{0}=\operatorname{Span}\left\{\partial_{z_{i}} \tilde{u}(0) / i=1, \ldots, d\right\}, \text { where } d:=\operatorname{dim}\left(K_{0}\right) .
\end{array}\right\}
$$

Condition $(B E)$ (for Bianchi-Egnell type condition) is a standard and natural assumption in the finite-dimensional reduction. It is satisfied when $M=\mathbb{R}^{n}$ and $h \equiv 0$ (see the classical references Rey [23] and Bianchi-Egnell [3]), also for some sign-changing solutions (see the recent example of Musso-Wei [20]). We refer to Ambrosetti-Malchiodi [1] for an abstract general setting for the use of nondegenerate critical manifolds.

In case condition $(B E)$ holds, the manifold $\mathcal{M}$ is the nondegenerate critical manifold, and minimizing $J_{0 \mid \mathcal{M}}$ exactly amounts to minimizing $I_{0 \mid \mathcal{M}}$, which is a considerable simplification for our problem. However, in general, the Bianchi-Egnell condition $(B E)$ does not hold. It is even exceptional: in Section 7 we exhibit examples of degenerate minimizers $u_{0}$ that are isolated among solutions to (2), and therefore, the only possible critical manifold is $\left\{u_{0}\right\}$ and is degenerate (see Propositions 7.1 and (7.3). Therefore, the classical methods using nondegenerate critical manifolds (see again the monograph Ambrosetti-Malchiodi [1]) are ineffective here. We refer to Del Pino-Felmer [9, Jeanjean-Tanaka [16, Byeon-Jeanjean [5], and Dancer 8 for an analysis on $\mathbb{R}^{n}$ without condition $(B E)$ based on topological arguments.

Our aim in the present article is to develop a new method to deal with the absence of nondegenerate critical manifold (that is when the Bianchi-Egnell condition $(B E)$ does not hold) by using analyticity. Indeed, due to our choice of the manifold $\mathcal{M}$, we are able to compare precisely all the terms in the analytic expansions of $I_{0}$ and $J_{0}$ on $\mathcal{M}$. As a consequence, we prove that the restriction of $J_{0}$ to $\mathcal{M}$ has a strict local minimum at $u_{0}$ if and only if $u_{0}$ is a strict local minimizer of $I_{0}$ (Theorem 6.1). This allows us to get a stable critical point for our problem.

This article is organized as follows. In Section 2, we state byproducts of our analysis. In Section 3, we define bubbles, we state the general construction theorem via finite-dimensional reduction and we recall existing results. In Section 4 , we perform a first Lyapunov-Schmidt reduction to construct the analytic manifold $\mathcal{M}$ of approximations of $u_{0}$. In Section 5, we reduce the proof of Theorem 1.1 to obtaining a stable well for $J_{0}$ restricted to $\mathcal{M}$. In Section [6] we use the analyticity to prove the equivalence of strict local minimization for $I_{0}$ and $J_{0}$ on $\mathcal{M}$. In Section 7 we construct examples of degenerate strict local minimizers.

Acknowledgement: the authors thank the referee for careful reading of this manuscript and useful remarks.

\section{Miscellaneous further Results}

Theorem 1.1 is a particular case of Theorem 2.1 below:

Theorem 2.1. Let $(M, g)$ be a compact Riemannian manifold of dimension $n \geq 3$. Let $h \in C^{0, \theta}(M)$ be such that $\Delta_{g}+h$ is coercive. Assume that there exists $u_{0} \in$ 
$C^{2, \theta}(M)$ that is a solution to (2) and a strict local minimizer of $I_{0}$. Assume that one of the following situations holds:

$$
\left\{\begin{array}{l}
3 \leq n \leq 5, \\
n=6 \text { and } c_{n} R_{g}-h<2 u_{0}, \\
3 \leq n \leq 9 \text { and } h \equiv c_{n} R_{g}, \\
n=10, h \equiv c_{n} R_{g} \text { and } u_{0}>\frac{5}{567} \mid \text { Weyl }\left.\left.\right|_{g}\right|_{g} ^{2}, \\
n \geq 3,(M, g) \text { is locally conformally flat and } h \equiv c_{n} R_{g} .
\end{array}\right\}
$$

Then there exist a solution of type $\left(u_{0}-B\right)$ to (1).

We are also in position to construct positive solutions in dimension $n=6$.

Theorem 2.2. Let $(M, g)$ be a smooth compact Riemannian manifold of dimension $n=6$ and let $h \in C^{0, \theta}(M)$ be such that $\Delta_{g}+h$ is coercive. Assume that there exists $u_{0} \in C^{2, \theta}(M)$ that is both a solution to (2) and an strict local minimizer of $I_{0}$. Assume that

$$
h-c_{6} R_{g}>2 u_{0}>0 \text { in } M .
$$

Then for $\varepsilon>0$ small, equation (11) admits a solution $u_{\varepsilon}>0$ such that $u_{\varepsilon}=$ $u_{0}+B_{\varepsilon}+o(1)$, where $\left(B_{\varepsilon}\right)_{\varepsilon}$ is a bubble and $\lim _{\varepsilon \rightarrow 0} o(1)=0$ in $H_{1}^{2}(M)$.

\section{Bubbles, General Existence theOrem AND PREliminary COMPutations}

This section essentially collects existing results from Robert-Vétois [24,25].

3.1. Bubbles. We follow the terminology in 25 . We say that $\left(B_{\varepsilon}\right)_{\varepsilon}$ is a bubble if there exists $\left(x_{\varepsilon}\right)_{\varepsilon} \in M$ and $\left(\mu_{\varepsilon}\right)_{\varepsilon} \in(0,+\infty)$ such that $\lim _{\varepsilon \rightarrow 0} \mu_{\varepsilon}=0$ and

$$
B_{\varepsilon}(x):=\left(\frac{\sqrt{n(n-2)} \mu_{\varepsilon}}{\mu_{\varepsilon}^{2}+d_{g}\left(x, x_{\varepsilon}\right)^{2}}\right)^{\frac{n-2}{2}} \text { for all } x \in M .
$$

There exists $r_{0} \in\left(0, i_{g}(M)\right)$ and $\Lambda \in C^{\infty}(M \times M)$ such that $(\xi, x) \mapsto \Lambda_{\xi}(x)>0$, $\Lambda_{\xi}(\xi)=1$ and :

(i) If $(M, g)$ is locally conformally flat (lcf), then $g_{\xi}=\Lambda_{\xi}^{4 /(n-2)} g$ is flat in $B_{\xi}\left(r_{0}\right)$.

(ii) If $(M, g)$ is not locally conformally flat (non lcf) then $g_{\xi}:=\Lambda_{\xi}^{\frac{4}{n-2}} g$ satisfies $d v_{g_{\xi}}=\left(1+O\left(d_{g_{\xi}}(\xi, \cdot)^{n}\right)\right) d x$ in a geodesic normal chart. An immediate consequence is that $R_{g_{\xi}}(\xi)=\left|\nabla R_{g_{\xi}}(\xi)\right|_{g_{\xi}}=0$ and $\Delta_{g_{\xi}} R_{g_{\xi}}(\xi)=\frac{1}{6}\left|\operatorname{Weyl}_{g}(\xi)\right|_{g}^{2}$. Moreover, $\nabla \Lambda_{\xi}(\xi)=0$. This change of metric is due to Lee-Parker [19].

We let $\chi$ be a smooth cutoff function such that $0 \leq \chi \leq 1$ in $\mathbb{R}, \chi=1$ in $\left[-r_{0} / 2, r_{0} / 2\right]$, and $\chi=0$ in $\mathbb{R} \backslash\left(-r_{0}, r_{0}\right)$. For any $\kappa \in\{-1,1\}$, any positive real number $\delta$ and any point $\xi$ in $M$, we define the function $W_{\kappa, \delta, \xi}$ on $M$ by

$$
W_{\kappa, \delta, \xi}(x):=\kappa \chi\left(d_{g_{\xi}}(x, \xi)\right) \Lambda_{\xi}(x)\left(\frac{\sqrt{n(n-2)} \delta}{\delta^{2}+d_{g_{\xi}}(x, \xi)^{2}}\right)^{\frac{n-2}{2}},
$$

where $d_{g_{\xi}}$ is the geodesic distance on $M$ associated with the metric $g_{\xi}$, the exponential map is taken with respect to the same metric $g_{\xi}$. As one checks, for any family $\left(\delta_{\varepsilon}\right)_{\varepsilon} \in(0,+\infty)$ going to 0 as $\varepsilon \rightarrow 0$, there exists a bubble $\left(B_{\varepsilon}\right)_{\varepsilon}$ such that

$$
W_{\kappa, \delta_{\varepsilon}, \xi_{\varepsilon}}=\kappa B_{\varepsilon}+o(1)
$$


in $H_{1}^{2}(M)$ when $\varepsilon \rightarrow 0$. Bubbles like $W_{\kappa, \delta, \xi}$ with a modification of the metric were introduced by Lee-Parker for an alternate resolution of the Yamabe problem. Using these bubbles smoothly depending on $\xi$ for finite dimensional reduction was first used in the article [14] by the second author and his collaborators.

Notations: Here and in the sequel, $\left(\Delta_{g}+h\right)^{-1}$ denotes the inverse of the natural isometric isomorphism

$$
\begin{aligned}
& \Delta_{g}+h: H_{1}^{2}(M) \quad \rightarrow \quad\left(H_{1}^{2}(M)\right)^{\prime} \\
& \phi \quad \mapsto \quad\left(\tau \mapsto \int_{M}\left((\nabla \phi, \nabla \tau)_{g}+h \phi \tau\right) d v_{g}\right) .
\end{aligned}
$$

Any function $f \in L^{\frac{2 n}{n+2}}(M)=\left(L^{2^{\star}}(M)\right)^{\prime}$ is seen as a linear form on $H_{1}^{2}(M)$. In the sequel $C$ will denote a constant independent of $\xi, \delta, \varphi, \varepsilon$. The value of $C$ can change from one line to the other for simplicity.

3.2. General existence theorem. For any $\nu_{0}>0$ and $\varepsilon>0$, we define

$$
\mathcal{D}_{\varepsilon}\left(\nu_{0}\right):=\left\{(\delta, \xi) \in\left(0, \nu_{0}\right) \times M /\left|\delta^{\varepsilon}-1\right|<\nu_{0}\right\} .
$$

We define for $\epsilon \in\left[0,2^{\star}-2\right)$

$$
J_{\varepsilon}(u):=\frac{1}{2} \int_{M}\left(|\nabla u|_{g}^{2}+h u^{2}\right) d v_{g}-\frac{1}{2^{\star}-\varepsilon} \int_{M}|u|^{2^{\star}-\varepsilon} d v_{g}=\frac{1}{2}\|u\|_{h}^{2}-F_{\varepsilon}(u)
$$

for all $u \in H_{1}^{2}(M)$, where

$$
\|u\|_{h}^{2}=(u, u)_{h}=\int_{M}\left(|\nabla u|_{g}^{2}+h u^{2}\right) d v_{g} \text { and } F_{\varepsilon}(u):=\frac{1}{2^{\star}-\varepsilon} \int_{M} H(u)^{2^{\star}-\varepsilon} d v_{g} .
$$

Here, $H(u):=|u|$ if $\kappa=-1$ and $H(u):=u_{+}$if $\kappa=1$. For any closed subspace $L \subset$ $H_{1}^{2}(M), \Pi_{L}$ will denote the orthogonal projection onto $L$ and $L^{\perp}$ the orthogonal complement of $L$ with respect to the Hilbert structure $(\cdot, \cdot)_{h}$.

We let $u \in C^{1}\left(B_{\nu_{0}}(0) \subset K_{0}, H_{1}^{2}(M)\right)$ be such that $u(0)=u_{0}$ and

$$
\left|\operatorname{det}\left(\Pi_{K_{0}} \partial_{1} u(\varphi), \cdots, \Pi_{K_{0}} \partial_{d} u(\varphi)\right)\right| \geq c_{0} \prod_{i=1}^{d}\left\|\partial_{i} u(\varphi)\right\|_{H_{1}^{2}}
$$

for some $c_{0}>0$ and all $\varphi \in B_{\nu_{0}}(0) \subset K_{0}$. Here, $d:=\operatorname{dim}_{\mathbb{R}}\left(K_{0}\right)$ and derivatives refer to a fixed basis of $K_{0}$. The following existence theorem is a consequence of Theorem 1.1 in Robert-Vétois [25]:

Theorem 3.1. There exists $\nu_{0}>0$ and there exists $\phi_{\varepsilon} \in C^{1}\left(B_{\nu_{0}}(0) \times \mathcal{D}_{\varepsilon}\left(\nu_{0}\right), K_{0}^{\perp}\right)$ such that for all $\varphi \in B_{\nu_{0}}(0) \subset K_{0},(\delta, \xi) \in \mathcal{D}_{\varepsilon}\left(\nu_{0}\right)$, the function $u_{\varepsilon}(\varphi, \delta, \xi):=$ $u(\varphi)+W_{\kappa, \delta, \xi}+\phi_{\varepsilon}(\varphi, \delta, \xi)$ is a critical point for $J_{\varepsilon}$ if and only if $(\varphi, \delta, \xi)$ is a critical point of $(\varphi, \delta, \xi) \mapsto J_{\varepsilon}\left(u_{\varepsilon}(\varphi, \delta, \xi)\right)$. Moreover, $\left\|\phi_{\varepsilon}(\varphi, \delta, \xi)\right\|_{h} \leq C \cdot R_{\varepsilon}(\varphi, \delta, \xi)$ where

$$
R_{\varepsilon}(\varphi, \delta, \xi):=\left\|\Pi_{K_{\delta, \xi}^{\perp}}\left(u(\varphi)+W_{\kappa, \delta, \xi}-\left(\Delta_{g}+h\right)^{-1}\left(F_{\varepsilon}^{\prime}\left(u(\varphi)+W_{\kappa, \delta, \xi}\right)\right)\right)\right\|_{h} .
$$

The space $K_{\delta, \xi}$ is defined below.

The projection onto $K_{\delta, \xi}^{\perp}$ in the rest $R_{\varepsilon}(\varphi, \delta, \xi)$ follows from Subsection 5.3 in [25]. The function $\phi_{\varepsilon}$ is defined implicitely as follows: given $(\varphi, \delta, \xi) \in B_{\nu_{0}}(0) \times \mathcal{D}_{\varepsilon}\left(\nu_{0}\right)$, $\phi_{\varepsilon}(\varphi, \delta, \xi)$ is the sole element of $K_{\delta, \xi}^{\perp}$ such that

$$
\Pi_{K_{\delta, \xi}^{\perp}}\left(u_{\varepsilon}(\varphi, \delta, \xi)-\left(\Delta_{g}+h\right)^{-1}\left(F_{\varepsilon}^{\prime}\left(u_{\varepsilon}(\varphi, \delta, \xi)\right)\right)=0 .\right.
$$

The linear space $K_{\delta, \xi}$ is defined as

$$
K_{\delta, \xi}:=\operatorname{Span}\left\{\varphi, Z_{\delta, \xi}, Z_{\delta, \xi, X}, \varphi \in K_{0} \text { and } X \in T_{\xi} M\right\},
$$


where

$$
\begin{aligned}
& Z_{\delta, \xi}(x):=\chi\left(d_{g_{\xi}}(x, \xi)\right) \Lambda_{\xi}(x) \delta^{\frac{n-2}{2}} \frac{d_{g_{\xi}}(x, \xi)^{2}-\delta^{2}}{\left(\delta^{2}+d_{g_{\xi}}(x, \xi)^{2}\right)^{\frac{n}{2}}}, \\
& Z_{\delta, \xi, X}(x):=\chi\left(d_{g_{\xi}}(x, \xi)\right) \Lambda_{\xi}(x) \delta^{\frac{n}{2}} \frac{\left\langle\left(\exp _{\xi}^{g_{\xi}}\right)^{-1}(x), X\right\rangle_{g_{\xi}(\xi)}}{\left(\delta^{2}+d_{g_{\xi}}(x, \xi)^{2}\right)^{\frac{n}{2}}}
\end{aligned}
$$

for all $x \in M$.

3.3. Estimate of the error term. For simplicity, we will often write $W:=W_{\kappa, \delta, \xi}$ and $\phi:=\phi_{\varepsilon}(\varphi, \delta, \xi)$ in this section. It follows from [24, Sections 5 and 7], that

$$
\left\|F_{\varepsilon}^{\prime}(u(\varphi)+W)-F_{\varepsilon}^{\prime}(u(\varphi))-F_{\varepsilon}^{\prime}(W)\right\|_{H_{1}^{2}(M)^{\prime}} \leq C \cdot \varepsilon_{1}(\delta),
$$

$$
F_{\varepsilon}(u(\varphi)+W)-F_{\varepsilon}(u(\varphi))-F_{\varepsilon}(W)-F_{\varepsilon}^{\prime}(u(\varphi)) W-F_{\varepsilon}^{\prime}(W) u(\varphi)=O\left(\varepsilon_{2}(\delta)\right),
$$

and

$$
\begin{aligned}
& \left\|W-\left(\Delta_{g}+h\right)^{-1}\left(F_{\varepsilon}^{\prime}(W)\right)\right\|_{h} \\
& \leq C \cdot\left(\varepsilon \ln \frac{1}{\delta}+\varepsilon_{1}(\delta)+\mathbf{1}_{\{n \geq 7\}}\left\|h-c_{n} R_{g}\right\|_{\infty} \delta^{2}+\mathbf{1}_{\{n \geq 15 \text { and non lcf }\}} \delta^{4}\right),
\end{aligned}
$$

where

$$
\varepsilon_{1}(\delta):=\left\{\begin{array}{ll}
\delta^{\frac{n-2}{2}} & \text { if } n<6 \\
\delta^{2}\left(\ln \frac{1}{\delta}\right)^{\frac{2}{3}} & \text { if } n=6 \\
\delta^{\frac{n+2}{4}} & \text { if } n>6
\end{array}\right\} \text { and } \varepsilon_{2}(\delta):=\left\{\begin{array}{ll}
\delta & \text { if } n=3 \\
\delta^{2} \ln \frac{1}{\delta} & \text { if } n=4 \\
\delta^{\frac{n}{2}} & \text { if } n \geq 5
\end{array}\right\}
$$

Plugging (10) and (12) in (9) yields

$$
\begin{aligned}
& R_{\varepsilon}(\varphi, \delta, \xi) \leq C \cdot \| \Pi_{K_{\delta, \xi}^{\perp}}\left(u(\varphi)-\left(\Delta_{g}+h\right)^{-1}\left(F_{0}^{\prime}(u(\varphi))\right) \|_{h}\right. \\
& +O\left(\varepsilon \ln \frac{1}{\delta}+\varepsilon_{1}(\delta)+\mathbf{1}_{\{n \geq 7\}}\left\|h-c_{n} R_{g}\right\|_{\infty} \delta^{2}+\mathbf{1}_{\{n \geq 15 \text { and non lcf }\}} \delta^{4}\right) .
\end{aligned}
$$

3.4. First expansion of the energy $J_{\varepsilon}$. The Taylor expansion of $J_{\varepsilon}$, the control of $\phi_{\varepsilon}$ in Theorem 3.1 and the definition (9) of $R_{\varepsilon}(\varphi, \delta, \xi)$ yield

$$
\begin{aligned}
& J_{\varepsilon}(u(\varphi)+W+\phi) \\
= & J_{\varepsilon}(u(\varphi)+W)+\left(u(\varphi)+W-\left(\Delta_{g}+h\right)^{-1}\left(F_{\varepsilon}^{\prime}(u(\varphi)+W)\right), \phi\right)_{h}+O\left(\|\phi\|_{h}^{2}\right) \\
= & J_{\varepsilon}(u(\varphi)+W)+\left(\Pi_{K_{\delta, \xi}^{\perp}}\left(u(\varphi)+W-\left(\Delta_{g}+h\right)^{-1}\left(F_{\varepsilon}^{\prime}(u(\varphi)+W)\right)\right), \phi\right)_{h}+O\left(\|\phi\|_{h}^{2}\right) \\
= & J_{\varepsilon}(u(\varphi)+W)+O\left(R_{\varepsilon}(\varphi, \delta, \xi)^{2}\right) .
\end{aligned}
$$

It then follows from (11) and (13) that

$$
\begin{aligned}
& \text { (15) } J_{\varepsilon}(u(\varphi)+W+\phi)=J_{\varepsilon}(u(\varphi))+J_{\varepsilon}\left(W_{\kappa, \delta, \xi}\right) \\
& +\left(u(\varphi)-\left(\Delta_{g}+h\right)^{-1}\left(F_{\varepsilon}^{\prime}(u(\varphi))\right), W\right)_{h}-F_{\varepsilon}^{\prime}(W) u(\varphi)+O\left(R_{\varepsilon}(\varphi, \delta, \xi)^{2}+\varepsilon_{2}(\delta)\right) .
\end{aligned}
$$

Since $\varphi \mapsto u(\varphi)>0$ is $C^{1}, u(0)=u_{0}$ is a solution to (2), we get that

$$
\left(u(\varphi)-\left(\Delta_{g}+h\right)^{-1}\left(u(\varphi)^{2^{\star}-1-\varepsilon}\right), W\right)_{h}=f_{1}(\varphi, \xi) \delta^{\frac{n-2}{2}}+o\left(\delta^{\frac{n-2}{2}}\right)
$$


when $\delta, \varepsilon \rightarrow 0$ and $f_{1} \in C^{1}\left(B_{\nu_{0}}(0) \times M, \mathbb{R}\right)\left(B_{\nu_{0}}(0) \subset K_{0}\right)$ and $f_{1}(0, \xi)=0$ for all $\xi \in M$. It follows from [24] that

$$
F_{\varepsilon}^{\prime}(W) u(\varphi)=\frac{\kappa 2^{n} \omega_{n-1} K_{n}^{-n}}{n(n(n-2))^{\frac{n-2}{4}} \omega_{n}} u(\varphi)[\xi] \delta^{\frac{n-2}{2}}+O\left(\delta^{\frac{n-2}{2}}\left(o(1)+\left|\delta^{\varepsilon}-1\right|\right)\right)
$$

when $(\delta, \varepsilon) \rightarrow 0$. Here, $\omega_{k}$ is the volume of the canonical unit $k$-sphere in $\mathbb{R}^{k+1}$ and $K_{n}$ is the best constant of the Sobolev inequality $\|u\|_{2^{\star}} \leq K\|\nabla u\|_{2}$ in $\mathbb{R}^{n}$. Finally, expanding $J_{\varepsilon}(u(\varphi))$ with respect to $\varepsilon$ and collecting (15), (16) and (17) yield

$$
\begin{aligned}
& J_{\varepsilon}(u(\varphi)+W+\phi)=J_{0}(u(\varphi))+\varepsilon f_{2}(\varphi)+J_{\varepsilon}\left(W_{\kappa, \delta, \xi}\right) \\
& +\left(f_{1}(\varphi, \xi)-\frac{\kappa 2^{n} \omega_{n-1} K_{n}^{-n}}{n(n(n-2))^{\frac{n-2}{4}} \omega_{n}} u(\varphi)[\xi]\right) \delta^{\frac{n-2}{2}} \\
& \quad+O\left(R_{\varepsilon}(\varphi, \delta, \xi)^{2}+\varepsilon_{2}(\delta)+\delta^{\frac{n-2}{2}}\left(o(1)+\left|\delta^{\varepsilon}-1\right|\right)\right)+o(\varepsilon)
\end{aligned}
$$

when $\delta, \varepsilon \rightarrow 0$. Here, $f_{2} \in C^{1}\left(B_{\nu_{0}}(0) \subset K_{0}, \mathbb{R}\right)$

3.5. Expansion of $J_{\varepsilon}\left(W_{\kappa, \delta, \xi}\right)$. The following result was obtained in [24]: there exists $\beta_{n}>0$ such that

$$
\begin{gathered}
J_{\varepsilon}\left(W_{\kappa, \delta, \xi}\right)=\frac{K_{n}^{-n}}{n}\left(1-\beta_{n} \varepsilon-\frac{(n-2)^{2}}{4}\left(\delta^{\varepsilon}-1\right)\right)+O\left(\varepsilon \delta^{2}+\varepsilon^{2}+\left(\delta^{\varepsilon}-1\right)^{2}\right) \\
+O\left(\mathbf{1}_{\left\{n \leq 5 \text { or lcf } \delta^{n-2}\right)}\right. \\
+\frac{K_{n}^{-n}}{n}\left\{\begin{array}{cc}
O\left(\left\|h-c_{3} R_{g}\right\|_{C^{0, \theta}} \delta\right) & \text { if } n=3 \\
3\left(h-c_{4} R_{g}\right)(\xi) \delta^{2} \ln \frac{1}{\delta}+O\left(\left\|h-c_{4} R_{g}\right\|_{C^{0, \theta}} \delta^{2}\right) & \text { if } n=4 \\
\frac{2(n-1)}{(n-2)(n-4)}\left(h-c_{n} R_{g}\right)(\xi) \delta^{2}+O\left(\left\|h-c_{n} R_{g}\right\|_{C^{0, \theta}} \delta^{2+\theta}\right) & \text { if } n \geq 5
\end{array}\right\} \\
+\frac{K_{n}^{-n}}{n}\left\{\begin{array}{ll}
-\frac{1}{64}\left|\mathrm{Weyl}_{g}(\xi)\right|_{g}^{2} \delta^{4} \ln \frac{1}{\delta}+O\left(\delta^{4}\right) & \text { if } n=6 \text { and non lcf } \\
-\frac{1}{24(n-4)(n-6)}\left|\operatorname{Weyl}_{g}(\xi)\right|_{g}^{2} \delta^{4}+O\left(\delta^{5}\right) & \text { if } n \geq 7 \text { and non lcf }
\end{array}\right\} .
\end{gathered}
$$

\section{Suitable approximation of $u_{0}$ AND ANAlyticity}

In 24, the blowing-up solutions of type $\left(u_{0}-B\right)$ are directly modeled on a nondegenerate function $u_{0}$. When $u_{0}$ is degenerate, the kernel $K_{0}$ plays a role in the finite-dimensional reduction and we consider a manifold of functions around $u_{0}$ parametrized locally by $K_{0}$.

Proposition 4.1. There exist $\nu_{0}>0$ small and $\phi \in C^{1}\left(B_{\nu_{0}}(0) \subset K_{0}, K_{0}^{\perp}\right)$ such that for all $\varphi \in K_{0}$ and $\psi \in K_{0}^{\perp}$ satisfying $\|\varphi\|_{h},\|\psi\|_{h}<\nu_{0}$, we have that

$$
\Pi_{K_{0}^{\perp}}\left(u_{0}+\varphi+\psi-\left(\Delta_{g}+h\right)^{-1}\left(F_{0}^{\prime}\left(u_{0}+\varphi+\psi\right)\right)\right)=0 \Leftrightarrow \psi=\phi(\varphi) .
$$

In particular, $\phi$ vanishes up to order 1 at 0 . Moreover, taking $\nu_{0}$ smaller if necessary, $u_{0}+\varphi+\phi(\varphi) \in C^{2, \theta}(M)$ is positive for all $\varphi \in B_{\nu_{0}}(0)$ and $\phi: B_{\nu_{0}}(0) \rightarrow$ $C^{2, \theta}(M)$ is analytic with respect to the associated topologies. 
The analytic manifold of approximation is $\mathcal{M}:=\left\{u_{0}+\varphi+\phi(\varphi) / \varphi \in B_{\nu_{0}}(0) \subset K_{0}\right\}$. Proposition 4.1 is a particular case of a more general result. Some definitions and notations are required in order to state the general result. We fix $f \in C^{1}(\mathbb{R})$ and we assume that there exists $u_{0} \in C^{2, \theta}(M)$ such that

$$
\Delta_{g} u_{0}+h u_{0}=f\left(u_{0}\right) \text { in } M
$$

We define

$$
K_{0}:=\left\{\varphi \in C^{2, \theta}(M) / \Delta_{g} \varphi+h \varphi=f^{\prime}\left(u_{0}\right) \varphi\right\} .
$$

In the sequel, $K_{0}$ will be regarded as a subset of the Hilbert space $H_{1}^{2}(M)$. It follows from Fredholm's theory for Hilbert spaces that $K_{0}$ is of finite dimension $d \in \mathbb{N}$. We prove the following result in the spirit of Dancer [7]:

Proposition 4.2. We let $f \in C^{1}(\mathbb{R})$ and $u_{0} \in C^{2, \theta}(M)$ be a solution to (20). We let $K_{0}$ be as in (21). Then there exist $\nu>0$ and $\phi \in C^{1}\left(B_{\nu}(0) \subset K_{0}, K_{0}^{\perp} \cap C^{2, \theta}(M)\right)$ such that for all $\varphi \in B_{\nu}(0) \subset K_{0}$ and $\psi \in B_{\nu}(0) \subset K_{0}^{\perp}$,

$$
\Pi_{K_{0}^{\perp}}\left(u_{0}+\varphi+\psi-\left(\Delta_{g}+h\right)^{-1}\left(f\left(u_{0}+\varphi+\psi\right)\right)\right)=0 \Leftrightarrow \psi=\phi(\varphi) .
$$

Moreover, if $f$ is analytic on an open interval $I$ and $u_{0}(x) \in I$ for all $x \in M$, then $\phi$ is analytic around 0 .

As one checks, the function $x \mapsto|x|^{2^{\star}-2} x$ is $C^{1}$ on $\mathbb{R}$ and analytic on $(0,+\infty)$. Therefore Proposition 4.1 is a direct consequence of Proposition 4.2 .

Proof of Proposition 4.2. The first part of the statement is a direct application of the implicit function theorem and regularity theory. Since $M$ is compact and $u_{0}$ is continuous, it follows from the analyticity of $f$ that there exists $A, B>0$ such that

$$
\left|a_{k}\left(u_{0}(x)\right)\right| \leq A \cdot B^{k} \text { for all } k \geq 0 \text { and } x \in M,
$$

where

$$
f\left(u_{0}(x)+h\right)=\sum_{k=0}^{\infty} a_{k}\left(u_{0}(x)\right) h^{k} \text { for all } x \in M \text { and } h \in\left(-B^{-1}, B^{-1}\right) .
$$

Since $\phi$ is $C^{\infty}$ its differential vanishes at 0 , we write for any $L \geq 2$ that

$$
\phi(\varphi)=\sum_{l=2}^{L} P_{l}(\varphi)+o\left(\|\varphi\|^{L}\right) \text { when } \varphi \rightarrow 0
$$

where for all $l \geq 2$ and $\varphi \in B_{\nu}(0) \subset K_{0}, P_{l}(\varphi) \in K_{0}^{\perp}$ is a homogeneous polynomial of degree $l$. We set $P_{1}(\varphi):=\varphi \in K_{0}$. Therefore, for any $L \geq 1$, we have that

$$
f\left(u_{0}+\varphi+\phi(\varphi)\right)=\sum_{k=0}^{L} a_{k}\left(u_{0}\right)\left(\sum_{l=1}^{L} P_{l}(\varphi)\right)^{k}+o\left(\|\varphi\|^{L}\right)
$$

when $\varphi \rightarrow 0$. We write that

$$
\left(\sum_{i=1}^{L} X_{i}\right)^{k}=\sum_{j=0}^{\infty} Q_{k, L, j}\left(X_{1}, \ldots, X_{L}\right)
$$

where

$$
Q_{k, L, j}\left(X_{1}, \ldots, X_{L}\right):=\sum_{\sum_{1}^{L} r_{l}=k ; \sum_{1}^{L} l r_{l}=j} \frac{k !}{\prod_{l=1}^{L} r_{l} !} \prod_{l=1}^{L} X_{l}^{r_{l}} .
$$


Note that $Q_{k, L, j}\left(X_{1}, \ldots, X_{L}\right)=0$ when $j \notin[k, L k]$, so all the sums make sense. Therefore, for any $L \geq 2$, the term of degree $L$ in (22) is

$$
\Pi_{K_{0}^{\perp}}\left(P_{L}(\varphi)-\left(\Delta_{g}+h\right)^{-1}\left(\sum_{k=0}^{L} a_{k}\left(u_{0}\right) Q_{k, L, L}\left(P_{1}(\varphi), \ldots, P_{L}(\varphi)\right)\right)\right)=0
$$

for all $L \geq 2$. In the sum, the term for $k=0$ is 0 , and the term for $k=1$ is $a_{1}\left(u_{0}\right) P_{L}(\varphi)=f^{\prime}\left(u_{0}\right) P_{L}(\varphi)$. Therefore, we have that

$$
P_{L}(\varphi)=L_{0}^{-1} \Pi_{K_{0}^{\perp}}\left(\left(\Delta_{g}+h\right)^{-1}\left(\sum_{k=2}^{L} a_{k}\left(u_{0}\right) Q_{k, L, L}\left(P_{1}(\varphi), \ldots, P_{L}(\varphi)\right)\right)\right)
$$

for all $L \geq 2$, where $L_{0}: K_{0}^{\perp} \rightarrow K_{0}^{\perp}$ is the isomorphism given by

$$
L_{0}(\psi)=\Pi_{K_{0}^{\perp}}\left(\psi-\left(\Delta_{g}+h\right)^{-1}\left(f^{\prime}\left(u_{0}\right) \psi\right)\right) \text { for all } \psi \in K_{0}^{\perp} .
$$

Note that since $k, L \geq 2$, the right-hand side of (25) is independent of $P_{L}(\varphi)$. We fix $\alpha \in(0,1)$. It follows from elliptic theory that there exists $C>0$ depending on $(M, g), h$ and $f^{\prime}\left(u_{0}\right)$ such that

$$
\left\|P_{L}(\varphi)\right\|_{C^{1, \alpha}} \leq C\left\|\sum_{k=2}^{L} a_{k}\left(u_{0}\right) Q_{k, L, L}\left(P_{1}(\varphi), \ldots, P_{L}(\varphi)\right)\right\|_{\infty}
$$

for all $L \geq 2$. We fix $K \geq 2$. Summing (26) from $L=2$ to $K$, using (23), (24) and the nonnegativity of the coefficients of $Q_{k, L, L}$, we get that

$$
\begin{aligned}
\sum_{L=2}^{K}\left\|P_{L}(\varphi)\right\|_{C^{1, \alpha}} & \leq C \cdot A \sum_{k=2}^{K} \sum_{L=k}^{K} B^{k} Q_{k, L, L}\left(\left\|P_{1}(\varphi)\right\|_{\infty}, \ldots,\left\|P_{L}(\varphi)\right\|_{\infty}\right) \\
& \leq C \cdot A \sum_{k=2}^{K} \sum_{L=k}^{K} B^{k} Q_{k, K, L}\left(\left\|P_{1}(\varphi)\right\|_{\infty}, \ldots,\left\|P_{K}(\varphi)\right\|_{\infty}\right) \\
& \leq C \cdot A \sum_{k=2}^{K}\left(B \sum_{l=1}^{K}\left\|P_{l}(\varphi)\right\|_{\infty}\right)^{k} .
\end{aligned}
$$

We define

$$
h_{K}(t):=\sup _{\|\varphi\|_{\infty} \leq t} \sum_{L=2}^{K}\left\|P_{L}(\varphi)\right\|_{\infty} .
$$

It follows from (27) that

$$
t+h_{K}(t) \leq \frac{1}{2 B} \Rightarrow h_{K}(t) \leq 2 C \cdot A \cdot B^{2} \cdot\left(t+h_{K}(t)\right)^{2} .
$$

Therefore, since $h_{K}$ is continuous and non-decreasing, we get that

$$
t<\varepsilon_{0}:=\min \left(\frac{1}{4 B}, \frac{1}{16 A B^{2} C}\right) \Rightarrow h_{K}(t) \leq \varepsilon_{0} \text { for all } K \geq 2 .
$$

As a consequence, the series $\left(\sum_{L=2}^{\infty} P_{L}(\varphi)\right)$ converges uniformly on $B_{\varepsilon_{0} / 2}(0) \subset$ $K_{0}$ in the $C^{0, \alpha}$-norm. Inequality (27) yields the convergence in $C^{1, \alpha}(M)$. The characterization (22) then yields

$$
\phi(\varphi)=\sum_{l=2}^{\infty} P_{l}(\varphi) \text { for all } \varphi \in B_{\varepsilon_{0}}(0) \subset K_{0} .
$$


Elliptic theory yields convergence in $C^{2, \theta}(M)$. This proves analyticity.

5. Reduction of the problem to the analysis of $J_{0}\left(u_{0}+\varphi+\phi(\varphi)\right)$

From now on, we define:

$$
u(\varphi):=u_{0}+\varphi+\phi(\varphi)
$$

for all $\varphi \in B_{\nu_{0}}(0) \subset K_{0}$, where $\phi(\varphi)$ is defined in Proposition 4.1. In particular,

$$
\Pi_{K_{0}^{\perp}}\left(u(\varphi)-\left(\Delta_{g}+h\right)^{-1}\left(F_{0}^{\prime}(u(\varphi))\right)=0\right.
$$

for all $\varphi \in B_{\nu_{0}}(0) \subset K_{0}$. Since $d \phi_{0} \equiv 0$, it then follows from Proposition 4.1 that $u$ satisfies the hypothesis (8). For $0<a<b$ to be fixed later, we define

$$
\delta:=t \varepsilon^{\frac{2}{n-2}}
$$

for $t \in[a, b]$. We assume that

$$
\{3 \leq n \leq 6\} \text { or }\left\{h \equiv c_{n} R_{g} \text { and } 3 \leq n \leq 10\right\} \text { or }\left\{h \equiv c_{n} R_{g} \text { and lcf }\right\} .
$$

Taking into account the expressions (13), (14), (18), (19), and (28), we then get that

$$
\begin{array}{r}
J_{\varepsilon}(u(\varphi)+W+\phi)=J_{0}(u(\varphi))+\varepsilon f_{2}(\varphi)+\frac{K_{n}^{-n}}{n}\left(1-\beta_{n} \varepsilon+\frac{n-2}{2} \varepsilon \ln \frac{1}{\varepsilon}\right) \\
+\varepsilon \cdot \frac{K_{n}^{-n}}{n} \cdot\left(\frac{(n-2)^{2}}{4} \ln \frac{1}{t}+F(\varphi, \xi) t^{\frac{n-2}{2}}\right)+o(\varepsilon)
\end{array}
$$

when $\varepsilon \rightarrow 0$ uniformly with respect to $t \in[a, b]$. Here, $F \in C^{1}\left(B_{\nu_{0}}(0) \times M\right)$ and we have that

$$
\begin{aligned}
F(0, \xi)=-\kappa \frac{2^{n} \omega_{n-1} u_{0}(\xi)}{(n(n-2))^{\frac{n-2}{4}} \omega_{n}} \\
+ \begin{cases}\frac{2(n-1)}{(n-2)(n-4)}\left(h-c_{n} R_{g}\right)(\xi) & \text { if } n=6 \\
-\frac{1}{24(n-4)(n-6)}\left|\mathrm{Weyl}_{g}(\xi)\right|^{2} & \text { if } n=10 \text { and } h \equiv c_{n} R_{g} \\
0 & \text { otherwise. }\end{cases}
\end{aligned}
$$

The assumptions (4) (for $\kappa=-1$ ) and (5) (for $\kappa=1)$ then yield

$$
F(0, \xi)>0 \text { for all } \xi \in M \text {. }
$$

We define

$$
a:=\frac{1}{2}\left(\frac{n-2}{2 \min _{\xi \in M} F(0, \xi)}\right)^{\frac{2}{n-2}} \text { and } b:=2\left(\frac{n-2}{2 \min _{\xi \in M} F(0, \xi)}\right)^{\frac{2}{n-2}} .
$$

Since $u_{0}$ is a strict local minimizer of $I_{0}$, it follows from Theorem 6.1 of next section that there exists $\nu_{1} \in\left(0, \nu_{0} / 2\right)$ such that

$$
J_{0}(u(\varphi))>J_{0}\left(u_{0}\right) \text { for all } \varphi \in B_{2 \nu_{1}}(0) \backslash\{0\} .
$$

Due to compactness, for any $\varepsilon>0$, there exists $\left(\varphi_{\varepsilon}, t_{\varepsilon}, \xi_{\varepsilon}\right) \in \bar{B}_{\nu_{1}}(0) \times[a, b] \times M$ such that

$$
\begin{aligned}
\min _{(\varphi, t, \xi) \in \bar{B}_{\nu_{1}}(0) \times[a, b] \times M} J_{\varepsilon}(u(\varphi) & \left.+W_{\kappa, t \varepsilon^{\frac{2}{n-2}}, \xi}+\phi_{\varepsilon}\left(\varphi, t \varepsilon^{\frac{2}{n-2}}, \xi\right)\right) \\
& =J_{\varepsilon}\left(u\left(\varphi_{\varepsilon}\right)+W_{\kappa, t_{\varepsilon} \varepsilon^{\frac{2}{n-2}}, \xi_{\varepsilon}}+\phi_{\varepsilon}\left(\varphi_{\varepsilon}, t_{\varepsilon} \varepsilon^{\frac{2}{n-2}}, \xi_{\varepsilon}\right)\right) .
\end{aligned}
$$


It then follows from the Taylor expansion (29), the choice of $0<a<b$ and (30) that $t_{\varepsilon} \in(a, b)$ and $\varphi_{\varepsilon} \in B_{\nu_{1}}(0)$ for small $\varepsilon>0$. Moreover, we have that

$$
\lim _{\varepsilon \rightarrow 0} t_{\varepsilon}=\left(\frac{n-2}{2 \min _{\xi \in M} F(0, \xi)}\right)^{\frac{2}{n-2}} \text { and } \lim _{\varepsilon \rightarrow 0} \varphi_{\varepsilon}=0,
$$

and $\left(\xi_{\varepsilon}\right)_{\varepsilon>0}$ approaches the set of minimizers of $F(0, \cdot)$ when $\varepsilon>0$ is small. Therefore, since $\left(\varphi_{\varepsilon}, t_{\varepsilon}, \xi_{\varepsilon}\right)$ lies in the interior of the domain, it is a critical point for the minimizing functional, and therefore, $\left(\varphi_{\varepsilon}, t_{\varepsilon} \varepsilon^{\frac{2}{n-2}}, \xi_{\varepsilon}\right)$ is a critical point for

$$
(\varphi, \delta, \xi) \mapsto J_{\varepsilon}\left(u(\varphi)+W_{\kappa, \delta, \xi}+\phi_{\varepsilon}(\varphi, \delta, \xi)\right)
$$

It then follows from Theorem 3.1 that $u_{\varepsilon}:=u\left(\varphi_{\varepsilon}\right)+W_{\kappa, t_{\varepsilon} \varepsilon^{\frac{2}{n-2}}, \xi_{\varepsilon}}+\phi_{\varepsilon}\left(\varphi_{\varepsilon}, t_{\varepsilon} \varepsilon^{\frac{2}{n-2}}, \xi_{\varepsilon}\right)$ is a solution to

$$
\Delta_{g} u_{\varepsilon}+h u_{\varepsilon}=\left|u_{\varepsilon}\right|^{2^{\star}-2-\varepsilon} u_{\varepsilon} \text { in } M
$$

for $\varepsilon>0$ small, and in addition, due to (17) and the error control of $\phi_{\varepsilon}$ in Theorem 3.1, we have that

$$
u_{\varepsilon}=u_{0}+\kappa B_{\varepsilon}+o(1)
$$

in $H_{1}^{2}(M)$ when $\varepsilon \rightarrow 0$, where $B_{\varepsilon}$ is as in (6) with $\mu_{\varepsilon}:=t_{\varepsilon} \varepsilon^{\frac{2}{n-2}}$. This proves Theorems 2.1 and 2.2. and therefore Theorem 1.1 .

We are now left with proving Theorem 6.1

\section{EquiVAlENCE OF STRICT LOCAL MiNimizERS}

This section is devoted to the proof of the following:

Theorem 6.1. The function $u_{0}$ is a strict local minimizer of $I_{0}$ iff 0 is a strict local minimizer of $\varphi \mapsto J_{0}\left(u_{0}+\varphi+\phi(\varphi)\right)$.

The proof goes through four claims and uses the analyticity of $\varphi \mapsto \phi(\varphi)$.

Claim 6.1. There exists $\nu_{0}>0$ such that

$$
\left\|u_{0}+\varphi+\phi(\varphi)\right\|_{h}^{2}-\left\|u_{0}+\varphi+\phi(\varphi)\right\|_{2^{\star}}^{2^{\star}}=\sum_{L=3}^{\infty} A_{L}(\varphi)
$$

and

$$
\left\|u_{0}+\varphi+\phi(\varphi)\right\|_{2^{\star}}^{2^{\star}}=\left\|u_{0}\right\|_{2^{\star}}^{2^{\star}}-\frac{n}{2} \sum_{L=3}^{\infty} \frac{L-2}{L} A_{L}(\varphi)
$$

for $\varphi \in B_{\nu_{0}}(0) \subset K_{0}$, where for any $L \geq 3, A_{L}(\varphi)$ is a homogeneous polynomial of degree $L$.

Proof of Claim 6.1. We are going to compute the Taylor expansions of the two lefthand-sides and we will use the analyticity of $\varphi \mapsto \phi(\varphi)$ to prove Claim 6.1 We fix $N \geq 2$. It follows from (24) that

$$
\begin{aligned}
& \text { (31) }\left\|u_{0}+\varphi+\phi(\varphi)\right\|_{2^{\star}}^{2^{\star}}=\int_{M}\left(u_{0}+\sum_{l=1}^{N} P_{l}(\varphi)\right)^{2^{\star}} d v_{g}+o\left(\|\varphi\|^{N}\right)=\left\|u_{0}\right\|_{2^{\star}}^{2^{\star}}+ \\
& \sum_{L=1}^{N} \sum_{j=1}^{L} \sum_{\sum_{l=1}^{L} r_{l}=j ; \sum_{l=1}^{L} l r_{l}=L} \frac{\prod_{i=0}^{j-1}\left(2^{\star}-i\right)}{\prod_{l=1}^{L} r_{l} !} \int_{M} u_{0}^{2^{\star}-j} \prod_{l=1}^{L} P_{l}(\varphi)^{r_{l}} d v_{g}+o\left(\|\varphi\|^{N}\right) .
\end{aligned}
$$


We claim that

$$
u_{0} \in K_{0}^{\perp} .
$$

We prove the claim. We let $\varphi$ be in $K_{0}$. The self-adjointness of the Laplacian yields

$$
\left(u_{0}, \varphi\right)_{h}=\int_{M}\left(\Delta_{g} u_{0}+h u_{0}\right) \varphi d v_{g}=\int_{M}\left(\Delta_{g} \varphi+h \varphi\right) u_{0} d v_{g} .
$$

It then follows from equation (2) and the definition (3) of $K_{0}$ that $\left(u_{0}, \varphi\right)_{h}=0$. This proves the claim.

It follows from (32) that the term for $L=1$ in (31) is $2^{\star} \int_{M} u_{0}^{2^{\star}-1} \varphi d v_{g}=0$. Separating the cases $j=1$ and $j \geq 2$, we get that

$$
\begin{aligned}
& \left\|u_{0}+\varphi+\phi(\varphi)\right\|_{2^{\star}}^{2^{\star}} \\
& =\left\|u_{0}\right\|_{2^{\star}}^{2^{\star}}+\sum_{L=2}^{N} \sum_{j=2}^{L} \sum_{\sum_{l} r_{l}=j ; \sum_{l} l r_{l}=L} \frac{\prod_{i=0}^{j-1}\left(2^{\star}-i\right)}{\prod_{l=1}^{L} r_{l} !} \int_{M} u_{0}^{2^{\star}-j} \prod_{l=1}^{L} P_{l}(\varphi)^{r_{l}} d v_{g} \\
& +2^{\star} \sum_{L=2}^{N} \int_{M} u_{0}^{2^{\star}-1} P_{L}(\varphi) d v_{g}+o\left(\|\varphi\|^{N}\right) .
\end{aligned}
$$

For $L \geq 2$, it follows from the expression (25) of $P_{L}(\varphi)$ that

$$
\left(L_{0} P_{L}(\varphi), u_{0}\right)_{h}=\sum_{j=2}^{L} \sum_{\sum_{l} r_{l}=j ; \sum_{l} l r_{l}=L} \frac{\prod_{i=1}^{j}\left(2^{\star}-i\right)}{\prod_{l=1}^{L} r_{l} !} \int_{M} u_{0}^{2^{\star}-j} \prod_{l=1}^{L} P_{l}(\varphi)^{r_{l}} d v_{g} .
$$

Since the operator $L_{0}$ is symmetric, we have that

$$
\left(L_{0} P_{L}(\varphi), u_{0}\right)_{h}=\left(P_{L}(\varphi), L_{0} u_{0}\right)_{h}=-\left(2^{\star}-2\right) \int_{M} u_{0}^{2^{\star}-1} P_{L}(\varphi) d v_{g} .
$$

Plugging into (33) the expression of $\int_{M} u_{0}^{2^{\star}-1} P_{L}(\varphi) d v_{g}$ obtained by combining (35) and (34), we get that

(36) $\left\|u_{0}+\varphi+\phi(\varphi)\right\|_{2^{\star}}^{2^{\star}}=\left\|u_{0}\right\|_{2^{\star}}^{2^{\star}}+$

$\frac{n}{2} \sum_{L=2}^{N} \sum_{j=2}^{L} \sum_{\sum_{l} r_{l}=j ; \sum_{l} l r_{l}=L} \frac{(j-2) \prod_{i=1}^{j-1}\left(2^{\star}-i\right)}{\prod_{l=1}^{L} r_{l} !} \int_{M} u_{0}^{2^{\star}-j} \prod_{l=1}^{L} P_{l}(\varphi)^{r_{l}} d v_{g}+o\left(\|\varphi\|^{N}\right)$.

Note that the term in the above sum vanishes for $j=2$. As one checks, for any $3 \leq j \leq L$, we have that

$$
\begin{aligned}
& \sum_{q=1}^{L-1} \frac{L-2 q}{L} \sum_{\sum_{l} s_{l}=j-1 ; \sum_{l} l s_{l}=L-q} \frac{1}{\prod_{l} s_{l} !} \int_{M} u_{0}^{2^{\star}-j}\left(\prod_{l} P_{l}(\varphi)^{s_{l}}\right) P_{q}(\varphi) d v_{g} \\
& =\sum_{q=1}^{L-1} \frac{L-2 q}{L} \sum_{\sum_{l} r_{l}=j ; \sum_{l} l r_{l}=L} \frac{r_{q}}{\prod_{l} r_{l} !} \int_{M} u_{0}^{2^{\star}-j} \prod_{l} P_{l}(\varphi)^{r_{l}} d v_{g} \\
& =\sum_{\sum_{l} r_{l}=j ; \sum_{l} l r_{l}=L}\left(\sum_{q=1}^{L-1} \frac{L-2 q}{L} r_{q}\right) \frac{1}{\prod_{l} r_{l} !} \int_{M} u_{0}^{2^{\star}-j} \prod_{l} P_{l}(\varphi)^{r_{l}} d v_{g} \\
& =(j-2) \sum_{\sum_{l} r_{l}=j ; \sum_{l} l r_{l}=L} \frac{1}{\prod_{l} r_{l} !} \int_{M} u_{0}^{2^{\star}-j} \prod_{l} P_{l}(\varphi)^{r_{l}} d v_{g} .
\end{aligned}
$$


Plugging this identity into (36) yields

$$
\left\|u_{0}+\varphi+\phi(\varphi)\right\|_{2^{\star}}^{2^{\star}}=\left\|u_{0}\right\|_{2^{\star}}^{2^{\star}}+\frac{n}{2} \sum_{L=3}^{N} \sum_{q=1}^{L-2} \frac{L-2 q}{L} u_{L-q, q}(\varphi)+o\left(\|\varphi\|^{N}\right),
$$

where

$$
\begin{aligned}
u_{k, q}(\varphi):=\sum_{j=2}^{k}( & \left.\prod_{i=1}^{j}\left(2^{\star}-i\right)\right) \\
& \times \sum_{\sum_{l} s_{l}=j ; \sum_{l} l s_{l}=k} \frac{1}{\prod_{l} s_{l} !} \int_{M} u_{0}^{2^{\star}-1-j}\left(\prod_{l} P_{l}(\varphi)^{s_{l}}\right) P_{q}(\varphi) d v_{g} .
\end{aligned}
$$

For any $L, q$ such that $q \geq 2$ and $L-q \geq 2$, the self-adjointness of $L_{0}$ yields $\left(L_{0} P_{q}(\varphi), P_{L-q}(\varphi)\right)_{h}=\left(P_{q}(\varphi), L_{0} P_{L-q}(\varphi)\right)_{h}$. Taking the explicit expression of (25) then yields

$$
u_{L-q, q}(\varphi)=u_{q, L-q}(\varphi) \text { for } 2 \leq q \leq L-2 .
$$

Therefore, for $L \geq 4$, we get that

$$
\sum_{q=2}^{L-2} \frac{L-2 q}{L} u_{L-q, q}(\varphi)=0,
$$

and then (37) yields

$$
\left\|u_{0}+\varphi+\phi(\varphi)\right\|_{2^{\star}}^{2^{\star}}=\left\|u_{0}\right\|_{2^{\star}}^{2^{\star}}+\frac{n}{2} \sum_{L=3}^{N} \frac{L-2}{L} u_{L-1,1}(\varphi)+o\left(\|\varphi\|^{N}\right) .
$$

We now estimate $\left\|u_{0}+\varphi+\phi(\varphi)\right\|_{h}^{2}-\left\|u_{0}+\varphi+\phi\right\|_{2^{\star}}^{2^{\star}}$. Using (22) and that $u_{0}, \phi(\varphi) \in$ $K_{0}^{\perp}$ for all $\varphi \in K_{0}$, we get that (writing $\phi=\phi(\varphi)$ for simplicity)

$$
\begin{aligned}
\| u_{0} & +\varphi+\phi\left\|_{h}^{2}-\right\| u_{0}+\varphi+\phi \|_{2^{\star}}^{2^{\star}} \\
& =\left(u_{0}+\varphi+\phi, u_{0}+\varphi+\phi-\left(\Delta_{g}+h\right)^{-1}\left(u_{0}+\varphi+\phi\right)^{2^{\star}-1}\right)_{h} \\
& =\left(\Pi_{K_{0}}\left(u_{0}+\varphi+\phi\right), \Pi_{K_{0}}\left(u_{0}+\varphi+\phi-\left(\Delta_{g}+h\right)^{-1}\left(u_{0}+\varphi+\phi\right)^{2^{\star}-1}\right)\right)_{h} \\
& \left.=\left(\varphi, \varphi-\left(\Delta_{g}+h\right)^{-1}\left(u_{0}+\varphi+\phi\right)^{2^{\star}-1}\right)\right)_{h} \\
& =\|\varphi\|_{h}^{2}-\int_{M}\left(u_{0}+\varphi+\phi\right)^{2^{\star}-1} \varphi d v_{g} .
\end{aligned}
$$

We fix $N \geq 3$ and write $\phi(\varphi)=\sum_{L=2}^{N-1} P_{l}(\varphi)+o\left(\|\varphi\|^{N-1}\right)$ when $\varphi \rightarrow 0$. A Taylor expansion and (24) yield

$$
\begin{aligned}
& \text { (40) } \int_{M}\left(u_{0}+\varphi+\phi(\varphi)\right)^{2^{\star}-1} \varphi d v_{g} \\
& =\int_{M} u_{0}^{2^{\star}-1} \varphi d v_{g}+\left(2^{\star}-1\right) \int_{M} u_{0}^{2^{\star}-2} \varphi^{2} d v_{g}+\sum_{l=2}^{N-1}\left(2^{\star}-1\right) \int_{M} u_{0}^{2^{\star}-2} \varphi P_{l}(\varphi) d v_{g} \\
& +\sum_{L=3}^{N} \sum_{j=2}^{L-1}\left(\prod_{i=1}^{j}\left(2^{\star}-i\right)\right)_{\sum_{l} s_{l}=j ; \sum_{l} l s_{l}=L-1} \frac{1}{\prod_{l} s_{l} !} \int_{M} u_{0}^{2^{\star}-1-j}\left(\prod_{l} P_{l}(\varphi)^{s_{l}}\right) \varphi d v_{g} \\
& +o\left(\|\varphi\|^{N}\right)
\end{aligned}
$$


FRÉDÉRIC ROBERT AND JÉRÔME VÉTOIS

when $\varphi \rightarrow 0$. The definition (3) of $K_{0}$ yields

$$
\left(2^{\star}-1\right) \int_{M} u_{0}^{2^{\star}-2} \varphi^{2} d v_{g}=\|\varphi\|_{h}^{2} .
$$

Moreover, since $P_{l}(\varphi) \in K_{0}^{\perp}$ for all $l \geq 2$, we get that

$$
\sum_{l=2}^{N-1}\left(2^{\star}-1\right) \int_{M} u_{0}^{2^{\star}-2} \varphi P_{l}(\varphi) d v_{g}=\left(\sum_{l=2}^{N-1} P_{l}(\varphi), \varphi\right)_{h}=0 .
$$

Plugging together (32) and (39)-(42) yields

$$
\left\|u_{0}+\varphi+\phi(\varphi)\right\|_{h}^{2}-\left\|u_{0}+\varphi+\phi(\varphi)\right\|_{2^{\star}}^{2^{\star}}=-\sum_{L=3}^{N} u_{L-1,1}(\varphi)+o\left(\|\varphi\|^{N}\right)
$$

when $\varphi \rightarrow 0$. We define

$$
=-\sum_{j=2}^{L-1}\left(\prod_{i=1}^{j}\left(2^{\star}-i\right)\right)_{\sum_{l} s_{l}=j ; \sum_{l} l s_{l}=L-1} \frac{1}{\prod_{l} s_{l} !} \int_{M} u_{0}^{2^{\star}-1-j}\left(\prod_{l} P_{l}(\varphi)^{s_{l}}\right) \varphi d v_{g}
$$

which is a homogenous polynomial of degree $L$. Claim 6.1 then follows from (38), (39), (43) and the analyticity of $\varphi \mapsto \phi(\varphi)$ (see Proposition 4.2).

We define

$$
\mathcal{S}_{K_{0}}:=\left\{\varphi \in K_{0} /\|\varphi\|_{h}=1\right\} .
$$

For any $\varphi \in \mathcal{S}_{K_{0}}$ and any $t \in\left(-\nu_{0}, \nu_{0}\right)$, we define

$$
f_{\varphi}(t):=\frac{J_{0}\left(u_{0}+t \varphi+\phi(t \varphi)\right)-J_{0}\left(u_{0}\right)}{t^{2} \cdot\left\|u_{0}\right\|_{h}^{2}} \text { if } t \neq 0 \text { and } f_{\varphi}(0)=0 .
$$

It follows from Claim 6.1 that $f_{\varphi}$ is analytic on $\left(-\nu_{0}, \nu_{0}\right)$ and that

$$
\left\|u_{0}+t \varphi+\phi(t \varphi)\right\|_{2^{\star}}^{2^{\star}}=\left\|u_{0}\right\|_{2^{\star}}^{2^{\star}}\left(1-\frac{n}{2} t^{3} f_{\varphi}^{\prime}(t)\right)
$$

for $|t|<\nu_{0}$. Therefore, we have that

$$
\begin{aligned}
I_{0}\left(u_{0}+t \varphi\right. & +\phi(t \varphi)) \\
& =I_{0}\left(u_{0}\right)\left(1+2 t^{2} f_{\varphi}(t)-\frac{n-2}{2} t^{3} f_{\varphi}^{\prime}(t)\right) \cdot\left(1-\frac{n}{2} t^{3} f_{\varphi}^{\prime}(t)\right)^{-\frac{2}{2^{\star}}} .
\end{aligned}
$$

Claim 6.2. We assume that $u_{0}$ is a strict local minimizer of $I_{0}$. Then there exists $\nu_{1} \in\left(0, \nu_{0}\right)$ such that for any $\varphi \in \mathcal{S}_{K_{0}}$ and $t \in\left(-\nu_{1}, \nu_{1}\right) \backslash\{0\}$, there holds

$$
\begin{aligned}
& f_{\varphi}(t)=0 \Rightarrow f_{\varphi}^{\prime}(t) \neq 0 \\
& f_{\varphi}^{\prime}(t)=0 \Rightarrow f_{\varphi}(t)>0 .
\end{aligned}
$$

Proof of Claim 6.2. If $f_{\varphi}(t)=f_{\varphi}^{\prime}(t)=0$, it then follows from (45) that $u_{0}+t \varphi+$ $\phi(t \varphi)$ is a minimizer for $I_{0}$ close to $u_{0}$, and therefore there exists $\lambda_{t}>0$ such that $u_{0}+t \varphi+\phi(t \varphi)=\lambda_{t} \cdot u_{0}$ for $t$ small. It then follows from the definition (22) of $\phi(t \varphi)$ that $\lambda_{t}=1$ and that $t \varphi=0$, which is a contradiction since $t \neq 0$ and $\varphi \neq 0$. Therefore $f_{\varphi}(t)$ and $f_{\varphi}^{\prime}(t)$ cannot vanish simultaneously for $t \neq 0$. Moreover, if $f_{\varphi}^{\prime}(t)=0$, (45) yields $f_{\varphi}(t) \geq 0$. Combining these assertions yields Claim 6.2. 
Claim 6.3. We assume that $u_{0}$ is a strict local minimizer of $I_{0}$. We claim that for all $\varphi \in \mathcal{S}_{K_{0}}$, there exists $\tilde{t}_{\varphi} \in\left(0, \nu_{1}\right)$ such that $f_{\varphi}(t)>0$ for all $t \in\left(0, \tilde{t}_{\varphi}\right)$.

Proof of Claim 6.3. It follows from Claim 6.2 that $f_{\varphi}$ does not vanish identically. Since it is analytic, there exists $a \neq 0$ and $k \geq 1$ (both depending on $\varphi$ ) such that $f_{\varphi}(t)=a t^{k}+o\left(t^{k}\right)$ when $t \rightarrow 0$. Obtaining from this the expansion of $f_{\varphi}^{\prime}(t)$ and plugging these expressions into (45) yield

$$
I_{0}\left(u_{0}+t \varphi+\phi(t \varphi)\right)=I_{0}\left(u_{0}\right)\left(1+2 a t^{k+2}+o\left(t^{k+2}\right)\right)
$$

when $t \rightarrow 0$. Since $u_{0}$ is a local minimizer, we get that $a \geq 0$, and then $a>0$. This yields the existence of $\tilde{t}_{\varphi}$. This proves Claim 6.3.

It follows from Claims 6.2 and 6.3 that for any $\varphi \in \mathcal{S}_{K_{0}}$, there exists $t_{\varphi} \in\left(0, \nu_{1}\right]$ such that $f_{\varphi}(t)>0$ for all $t \in\left(0, t_{\varphi}\right)$, and in case $t_{\varphi}<\nu_{1}$, we have that $f_{\varphi}(t)<0$ for all $t \in\left(t_{\varphi}, \nu_{1}\right)$.

Claim 6.4. We assume that $u_{0}$ is a strict local minimizer of $I_{0}$. We claim that there exists $\nu_{2}>0$ such that $t_{\varphi}>\nu_{2}$ for all $\varphi \in \mathcal{S}_{K_{0}}$.

Proof of Claim 6.4. We prove Claim 6.4 by contradiction. Indeed, otherwise, there exists a sequence $\left(\varphi_{i}\right) \in \mathcal{S}_{K_{0}}$ such that $t_{\varphi_{i}} \rightarrow 0$ when $i \rightarrow+\infty$ and $f_{\varphi_{i}}\left(t_{\varphi_{i}}\right)=0$ for all $i$. Up to a subsequence, we can assume that $\varphi_{i} \rightarrow \varphi \in \mathcal{S}_{K_{0}}$ when $i \rightarrow+\infty$. We fix $t \in\left(0, \nu_{1}\right)$. Then for $i$ large enough, we have $t_{\varphi_{i}}<t$, and therefore $f_{\varphi_{i}}(t)<0$. Passing to the limit when $i \rightarrow+\infty$ yields $f_{\varphi}(t) \leq 0$ for all $t \in\left(0, \nu_{1}\right)$. This is a contradiction with Claim 6.3 This proves Claim 6.4

Proof of Theorem 6.1, first implication: We assume that $u_{0}$ is a strict local minimizer of $I_{0}$. It follows from Claim 6.4 that $J_{0}\left(u_{0}+\varphi+\phi(\varphi)\right)>J_{0}\left(u_{0}\right)$ for all $\varphi \in B_{\nu_{2}}(0) \backslash\{0\}$. This proves the first implication of Theorem 6.1.

Proof of Theorem 6.1, second implication: We assume that there exists $\nu_{1}>0$ such that $J_{0}\left(u_{0}+\varphi+\phi(\varphi)\right)>J_{0}\left(u_{0}\right)$ for all $\varphi \in B_{\nu_{1}}(0) \backslash\{0\}$. For $\varphi \in B_{\nu_{1}}(0)$, we define $\delta A(\varphi)$ and $\delta B(\varphi)$ such that

$\left\|u_{0}+\varphi+\phi(\varphi)\right\|_{h}^{2}=\left\|u_{0}\right\|_{h}^{2} \cdot(1+\delta A(\varphi))$ and $\left\|u_{0}+\varphi+\phi(\varphi)\right\|_{2^{\star}}^{2^{\star}}=\left\|u_{0}\right\|_{h}^{2} \cdot(1+\delta B(\varphi))$.

Therefore, we have that

$$
\begin{aligned}
& J_{0}\left(u_{0}+\varphi+\phi(\varphi)\right)=J_{0}\left(u_{0}\right)+\left\|u_{0}\right\|_{h}^{2} \cdot\left(\frac{1}{2} \delta A(\varphi)-\frac{1}{2^{\star}} \delta B(\varphi)\right), \\
& I_{0}\left(u_{0}+\varphi+\phi(\varphi)\right)=I_{0}\left(u_{0}\right) \cdot(1+\delta A(\varphi))(1+\delta B(\varphi))^{-2 / 2^{\star}}
\end{aligned}
$$

for all $\varphi \in B_{\nu_{1}}(0)$. It follows from our assumption and (46) that $\delta A(\varphi)>\frac{2}{2^{\star}} \delta B(\varphi)$ for all $\varphi \in B_{\nu_{1}}(0) \backslash\{0\}$. It then follows from (47) that

$$
I_{0}\left(u_{0}+\varphi+\phi(\varphi)\right)>I_{0}\left(u_{0}\right) \text { for all } \varphi \in B_{\nu_{1}}(0) \backslash\{0\} .
$$

We now let $\left(u_{i}\right) \in H_{1}^{2}(M)$ be minimizers for $I_{0}$ such that $\lim _{i \rightarrow+\infty} u_{i}=u_{0}$. It follows from regularity theory that $u_{i} \in C^{2, \theta}(M)$ for all $i$ and that the convergence holds in $C^{2, \theta}(M)$. Without loss of generality, we can assume that $u_{i}$ is a solution to (2) for all $i$. It then follows from the definition of $\phi$ (see Proposition 4.1) that there exists $\varphi_{i} \in K_{0}$ such that $u_{i}=u_{0}+\varphi_{i}+\phi\left(\varphi_{i}\right)$ for all $i$. Since $u_{i}$ is a local minimizer, it then follows from (48) that $\varphi_{i}=0$ for $i$ large, and thus $u_{i}=u_{0}$. Then $u_{0}$ is a strict local minimizer of $I_{0}$. This proves the second implication of Theorem6.1. 


\section{EXAMPLES}

In this section, we provide examples of strict local minimizers for the functional $I_{0}$, and hence for $J_{0}$ by Theorem 6.1. We let $u_{0} \in C^{2}(M)$ be a solution to (2). In particular $I_{0}^{\prime}\left(u_{0}\right)=0$. As a preliminary remark,

$$
\text { if } u_{0} \text { is a local minimizer of } I_{0} \text { then } I_{0}^{\prime \prime}\left(u_{0}\right) \geq 0 \text {. }
$$

Moreover, since $u_{0}$ is a solution to (2), the kernel of $I_{0}^{\prime \prime}\left(u_{0}\right)$ is given as follows: for any $f_{0} \in H_{1}^{2}(M)$,

$$
\left\{I_{0}^{\prime \prime}\left(u_{0}\right)\left(f_{0}, f\right)=0 \text { for all } f \in H_{1}^{2}(M)\right\} \Leftrightarrow\left\{f_{0} \in \mathbb{R} u_{0} \oplus K_{0}\right\} .
$$

Therefore, $I_{0}^{\prime \prime}\left(u_{0}\right)$ cannot be positive definite, and a specific analysis along $K_{0}$ is necessary. It follows from the expression (44) of $A_{L}(\varphi)$ that

$$
\begin{aligned}
A_{3}(\varphi)=- & \frac{\left(2^{\star}-1\right)\left(2^{\star}-2\right)}{2} \int_{M} u_{0}^{2^{\star}-3} \varphi^{3} d v_{g}, \\
A_{4}(\varphi)=- & \left(2^{\star}-1\right)\left(2^{\star}-2\right)\left(\int_{M} u_{0}^{2^{\star}-3} \varphi^{2} P_{2}(\varphi) d v_{g}\right. \\
& \left.+\frac{2^{\star}-3}{6} \int_{M} u_{0}^{2^{\star}-4} \varphi^{4} d v_{g}\right)
\end{aligned}
$$

for all $\varphi \in K_{0}$. Moreover, it follows from Claim 6.1 that

$$
I_{0}\left(u_{0}+\varphi+\phi(\varphi)\right)=I_{0}\left(u_{0}\right) \cdot\left(1+\frac{2 A_{3}(\varphi)}{3\left\|u_{0}\right\|_{2^{\star}}^{2^{\star}}}+\frac{A_{4}(\varphi)}{2\left\|u_{0}\right\|_{2^{\star}}^{2^{\star}}}+o\left(\|\varphi\|^{4}\right)\right)
$$

when $\varphi \rightarrow 0$. Therefore,

(54) if $u_{0}$ is a local minimizer of $I_{0}$ then $A_{3} \equiv 0$ and $A_{4}(\varphi) \geq 0$ for all $\varphi \in K_{0}$.

In the case of the Yamabe equation, this condition appeared in Kobayashi [18. Conversely, we have the following result:

Proposition 7.1. Assume that $A_{3} \equiv 0, I_{0}^{\prime \prime}\left(u_{0}\right) \geq 0$ and $A_{4}(\varphi)>0$ for all $\varphi \in$ $K_{0} \backslash\{0\}$. Then $u_{0}$ is a strict local minimizer for $I_{0}$. Moreover, there exists $\nu_{1}>0$ such that $u_{0}$ is the only solution to $\Delta_{g} u+h u=u^{2^{\star}-1}$ in $B_{\nu_{1}}\left(u_{0}\right)$.

Proof of Proposition 7.1. We begin with proving the first part of the proposition. We let $\varphi \in K_{0}$ and $\phi \in\left(\mathbb{R} u_{0} \oplus K_{0}\right)^{\perp}$ be in $H_{1}^{2}(M)$. A Taylor expansion yields

$$
\begin{aligned}
I_{0}\left(u_{0}+\varphi+\phi\right)=I_{0}\left(u_{0}\right. & +\varphi+\phi(\varphi))+I_{0}^{\prime}\left(u_{0}+\varphi+\phi(\varphi)\right)(\phi-\phi(\varphi)) \\
& +\frac{1}{2} I_{0}^{\prime \prime}\left(u_{0}\right)(\phi-\phi(\varphi), \phi-\phi(\varphi))+o\left(\|\phi-\phi(\varphi)\|_{h}^{2}\right)
\end{aligned}
$$

as $\varphi, \phi \rightarrow 0$. Since $\phi \in\left(\mathbb{R} u_{0} \oplus K_{0}\right)^{\perp}, \phi(\varphi) \in K_{0}^{\perp}$ and $\phi(\varphi)=O\left(\|\varphi\|_{h}^{2}\right)$, we get that

$$
\|\phi-\phi(\varphi)\|_{h}^{2}=\left\|\Pi_{\left(\mathbb{R} u_{0} \oplus K_{0}\right)^{\perp}}(\phi-\phi(\varphi))\right\|_{h}^{2}+O\left(\|\varphi\|_{h}^{4}\right)
$$

as $\varphi, \phi \rightarrow 0$. As one can check, for any $u, v \in H_{1}^{2}(M), u \not \equiv 0$, we have that

$$
I_{0}^{\prime}(u)(v)=\frac{2 I_{0}(u)}{\|u\|_{h}^{2}}\left(u-\frac{\|u\|_{h}^{2}}{\int_{M}|u|^{2^{\star}} d v_{g}}\left(\Delta_{g}+h\right)^{-1}\left(F_{0}^{\prime}(u)\right), v\right)_{h} .
$$

Therefore, since $\phi-\phi(\varphi) \in K_{0}^{\perp}$, it follows from the definition of $\phi(\varphi)$ in Proposition 4.1. Claim 6.1 and $A_{3} \equiv 0$ that

$$
I_{0}^{\prime}\left(u_{0}+\varphi+\phi(\varphi)\right)(\phi-\phi(\varphi))=O\left(\|\varphi\|_{h}^{4}\|\phi-\phi(\varphi)\|_{h}\right)=o\left(\|\varphi\|_{h}^{4}\right)
$$


as $\varphi, \phi \rightarrow 0$. Since $I_{0}^{\prime \prime}\left(u_{0}\right) \geq 0$, it follows from (50) that there exists $c_{1}>0$ such that

$$
I_{0}^{\prime \prime}\left(u_{0}\right)(u, u) \geq 4 c_{1}\left\|\Pi_{\left(\mathbb{R} u_{0} \oplus K_{0}\right)^{\perp}}(u)\right\|_{h}^{2}
$$

for all $u \in H_{1}^{2}(M)$. The positivity of $A_{4}$ yields the existence of $c_{2}>0$ such that

$$
A_{4}(\varphi) \geq c_{2}\|\varphi\|^{4} \text { for all } \varphi \in K_{0} .
$$

Plugging (53), (56), (57), (58) and (59) into (55) yields the existence of $c_{3}>0$ such that

$$
I_{0}\left(u_{0}+\varphi+\phi\right) \geq I_{0}\left(u_{0}\right)+c_{3}\|\varphi\|^{4}+c_{1}\left\|\Pi_{\left(\mathbb{R} u_{0} \oplus K_{0}\right)^{\perp}}(\phi-\phi(\varphi))\right\|_{h}^{2}
$$

as $\varphi, \phi \rightarrow 0$, where $\varphi \in K_{0}$ and $\phi \in\left(\mathbb{R} u_{0} \oplus K_{0}\right)^{\perp}$. This proves that $u_{0}$ is a strict local minimizer of $I_{0}$.

For the second part, for any solution $u \in B_{\nu_{1}}\left(u_{0}\right)$, we decompose $u:=u_{0}+\varphi+\psi$ where $\varphi \in K_{0}$ and $\psi \in K_{0}^{\perp}$. We have that $\|\varphi\|<\nu_{1}$ and $\|\psi\|<\nu_{1}$. It follows from Proposition 4.2 that if $\nu_{1}>0$ is small enough, then $\psi=\phi(\varphi)$ and $u=u(\varphi)$. The positivity of $A_{4}$ yields the existence of $c>0$ such that $A_{4}(\varphi) \geq 2 c\|\varphi\|^{4}$ for all $\varphi \in K_{0}$. It then follows from Claim 6.1 that $\|u\|_{h}^{2}-\|u\|_{2^{\star}}^{2^{\star}} \geq c\|\varphi\|^{4}$. Since $u$ is a solution to the equation, we then get that $\varphi=0$ and then $u=u_{0}$.

In this section, we exhibit situations in which the hypothesis of Proposition 7.1 hold, which yields strict local minimizers for $I_{0}$.

7.1. The expression of $A_{4}$ when $u_{0}$ is constant. We assume here that $h, u_{0}>0$ are positive constants. In particular, we have that $h=u_{0}^{2^{\star}-2}$ and that

$$
K_{0}=\left\{\varphi \in C^{2}(M) / \Delta_{g} \varphi=\lambda \varphi\right\}
$$

where $\lambda:=\left(2^{\star}-2\right) u_{0}^{2^{\star}-2}>0$. In other words, $u_{0}$ is degenerate if and only if $\lambda$ is an eigenvalue of $\Delta_{g}$. As one checks, the operator

$$
\begin{array}{rlc}
\Delta_{g}-\lambda: \quad K_{0}^{\perp} & \rightarrow & \left(K_{0}^{\perp}\right)^{\prime} \\
\phi & \mapsto \quad\left(\tau \mapsto \int_{M}\left((\nabla \phi, \nabla \tau)_{g}-\lambda \phi \tau\right) d v_{g}\right)
\end{array}
$$

is a bi-continuous isomorphism and then definition (25) yields

$$
P_{2}(\varphi)=\frac{\left(2^{\star}-1\right)\left(2^{\star}-2\right)}{2}\left(\Delta_{g}-\lambda\right)^{-1}\left(u_{0}^{2^{\star}-3} \varphi^{2}\right)
$$

for all $\varphi \in K_{0}$. As a consequence, the expression (52) of $A_{4}$ can be rewritten

$$
\begin{array}{r}
A_{4}(\varphi)=\left(2^{\star}-1\right)\left(2^{\star}-2\right) u_{0}^{2^{\star}-4}\left(-\frac{\left(2^{\star}-1\right) \lambda}{2} \int_{M} \varphi^{2}\left(\Delta_{g}-\lambda\right)^{-1}\left(\varphi^{2}\right) d v_{g}\right. \\
\left.-\frac{2^{\star}-3}{6} \int_{M} \varphi^{4} d v_{g}\right)
\end{array}
$$

for all $\varphi \in K_{0}$. 
7.2. The case of the Yamabe equation on the canonical sphere. In the case of the Yamabe equation on the sphere, the kernel $K_{0}$ parametrizes exactly the noncompact set of minimizers, which makes $A_{4}$ vanish. More precisely,

Proposition 7.2. [Kobayashi [18] Assume that $(M, g)=\left(\mathbb{S}^{n}\right.$, can) and that $h \equiv$ $c_{n} R_{\text {can }}$. Then any solution $u_{0}$ to (2) is minimizing and $A_{4} \equiv 0$ for all $u_{0}$.

Proof of Proposition 7.2. This result is a consequence of Theorem 2.1 in Kobayashi [18. We give here an independent proof for the sake of self-content. The vanishing of $A_{4}$ is a consequence of the direct computation in the proof of (ii) of Proposition 7.3 below. We give here a shorter and less technical proof that stresses on properties of solutions to the scalar curvature equation on the sphere

$$
\Delta_{\text {can }}+c_{n} R_{\text {can }} u=u^{2^{\star}-1} \text { in } \mathbb{S}^{n} .
$$

The proof relies on two facts: first, the elements of the kernel $K_{0}$ satisfy a BianchiEgnell condition; second, all solutions to (62) minimize $I_{0}$ (see Obata 21]).

We fix $\varphi \in K_{0}$. It follows from properties of the canonical sphere (see below) that there exists $t \in \mathbb{R} \mapsto u(t)$ a smooth function such that $u(t) \in C^{\infty}\left(\mathbb{S}^{n}\right)$ is a solution to (62) for all $t, u(0)=u_{0}$ and $u^{\prime}(0)=\varphi$. This is Bianchi-Egnell condition. Since $u(t)$ is a positive solution to (62), it follows from Proposition 4.1 that for $t$ small, there exists $\varphi(t) \in K_{0}$ such that $u(t)=u_{0}+\varphi(t)+\phi(\varphi(t))$. Moreover, $t \mapsto \varphi(t)$ is smooth, $\varphi(0)=0$ and $\varphi^{\prime}(0)=\varphi$. It follows from (54) that $A_{3} \equiv 0$ since $u_{0}$ minimizes $I_{0}$. It then follows from the expansion (53) of $A_{4}$ that

$$
\frac{A_{4}(\varphi)}{2\left\|u_{0}\right\|_{2^{\star}}^{2}}=\lim _{t \rightarrow 0} \frac{I_{0}\left(u_{0}+\varphi(t)+\phi(\varphi(t))\right)-I_{0}\left(u_{0}\right)}{t^{4} I_{0}\left(u_{0}\right)}=\lim _{t \rightarrow 0} \frac{I_{0}(u(t))-I_{0}\left(u_{0}\right)}{t^{4} I_{0}\left(u_{0}\right)} .
$$

Moreover, it follows from Obata 21 that positive solutions to (62) are all minimizing, and then $I_{0}(u(t))=I_{0}\left(u_{0}\right)$ for all small $t$. Therefore, we get that $A_{4}(\varphi)=0$ for all $\varphi \in K_{0}$.

We are now left with proving the existence of $t \mapsto u(t)$. Up to conformal transformation (see Obata [21]), we assume that $u_{0}$ is the sole positive constant solution to (62). In this case, $K_{0}=\left\{\varphi \in C^{2}\left(\mathbb{S}^{n}\right) / \Delta_{\operatorname{can}} \varphi=n \varphi\right\}$ is the space of first spherical harmonics. We fix $\varphi \in K_{0}$ and we let $Z:=\overrightarrow{\operatorname{grad}}(\varphi)$ be the associated vector field. This is a conformal vector field and, denoting $f_{t}$ the associated flow, we have that $f_{t}^{\star}$ can $=\omega(t)^{4 /(n-2)}$ can for some positive function $t \mapsto \omega(t) \in C^{\infty}\left(\mathbb{S}^{n}\right)$ such that $\omega(0)=1$. It follows from the conformal invariance of the scalar curvature equation that $u(t):=\omega(t) u_{0}$ is also a solution to (62) for all $t$. Moreover, since $f_{t}^{\star}$ can $=\omega(t)^{4 /(n-2)}$ can, we have that $\omega^{\prime}(0)=-\frac{n-2}{2 n} \Delta_{\text {can }} \varphi=\frac{n-2}{2 n} \operatorname{div}_{\text {can }}(Z)=-\frac{n-2}{2} \varphi$, and then $u^{\prime}(0)=c \varphi$ for some $c \neq 0$. This proves the result after rescaling.

7.3. Product of manifolds and examples of degenerate strict local minimizers. Let $\left(M_{1}, g_{1}\right)$ and $\left(M_{2}, g_{2}\right)$ be two compact manifolds of respective dimensions $d \geq 1$ and $n-d \geq 1$ with $n \geq 3$. We consider the Riemannian manifold $M:=M_{1} \times M_{2}$ endowed with the product metric $g:=g_{1} \oplus g_{2}$. For $i=1,2$, we let $\lambda_{1}\left(M_{i}, g_{i}\right)>0$ be the first nonzero eigenvalue of $\Delta_{g_{i}}$ on $M_{i}$. We define

$$
h:=\frac{\lambda_{1}\left(M_{1}, g_{1}\right)}{2^{\star}-2} \text { and } u_{0}:=\left(\frac{\lambda_{1}\left(M_{1}, g_{1}\right)}{2^{\star}-2}\right)^{\frac{n-2}{4}},
$$


so that $u_{0}$ is the only positive constant solution to $\Delta_{g} u_{0}+h u_{0}=u_{0}^{2^{\star}-1}$ in $M$. When $d \geq 3$, we define

$$
\tilde{h}:=\frac{\lambda_{1}\left(M_{1}, g_{1}\right)}{2_{d}^{\star}-2} \text { and } \tilde{u}_{0}:=\left(\frac{\lambda_{1}\left(M_{1}, g_{1}\right)}{2_{d}^{\star}-2}\right)^{\frac{d-2}{4}} \text {, where } 2_{d}^{\star}:=\frac{2 d}{d-2}
$$

so that $\tilde{u}_{0}$ is the only positive constant solution to

$$
\Delta_{g_{1}} \tilde{u}_{0}+\tilde{h} \tilde{u}_{0}=\tilde{u}_{0}^{2_{d}^{\star}-1} \text { in } M_{1} .
$$

In particular, $\tilde{u}_{0}$ is a critical point for the functional

$$
\tilde{I}_{0}(u):=\frac{\int_{M_{1}}\left(|\nabla u|_{g_{1}}^{2}+\tilde{h} u^{2}\right) d v_{g_{1}}}{\left(\int_{M_{1}}|u|^{2_{d}^{\star}} d v_{g_{1}}\right)^{\frac{2}{2 \star}}}
$$

for $u \in H_{1}^{2}\left(M_{1}\right) \backslash\{0\}$. We prove the following:

Proposition 7.3. Let $\left(M_{1}, g_{1}\right)$ and $\left(M_{2}, g_{2}\right)$ be two compact manifolds of respective dimensions $d \geq 1$ and $n-d \geq 1$ with $n \geq 3$. We consider the Riemannian manifold $M:=M_{1} \times M_{2}$ of dimension $n \geq 3$ endowed with the product metric $g:=g_{1} \oplus g_{2}$. We let $h, u_{0}>0$ be as in (63). We assume that one of the following cases hold:

(i) $d \geq 3, \lambda_{1}\left(M_{1}, g_{1}\right)<\lambda_{1}\left(M_{2}, g_{2}\right)$, and $\tilde{u}_{0}$ is a local minimizer of $\tilde{I}_{0}$,

(ii) $d \geq 1$ and $\left(M_{1}, g_{1}\right)=\left(\mathbb{S}^{d}(r)\right.$, can $)$ with $r>\sqrt{\frac{d}{\lambda_{1}\left(M_{2}, g_{2}\right)}}$.

Then $u_{0}$ is a degenerate solution to (2) and $I_{0}^{\prime \prime}\left(u_{0}\right) \geq 0$. Moreover, we have that $A_{3}(\varphi)=0$ and $A_{4}(\varphi)>0$ for all $\varphi \in K_{0} \backslash\{0\}$. In particular, $u_{0}$ is a strict local minimizer of $I_{0}$.

In the case of the Yamabe equation on the product of spheres, this proposition is a consequence of Kobayashi [18].

Proof of Proposition 7.3. We let $\left(M_{1}, g_{1}\right),\left(M_{2}, g_{2}\right)$ be as in the proposition. Since $\lambda_{1}\left(\mathbb{S}^{d}(r)\right.$, can $)=d r^{-2}$ (see Berger-Gauduchon-Mazet [2]), we have that

$$
\lambda_{1}\left(M_{1}, g_{1}\right)<\lambda_{1}\left(M_{2}, g_{2}\right)
$$

in both Cases (i) and (ii). As one checks,

$$
K_{0}=\left\{\varphi \in C^{2}(M) / \Delta_{g} \varphi=\lambda_{1}\left(M_{1}, g_{1}\right) \varphi\right\} .
$$

It follows from spectral theory for products that $K_{0}$ is spanned by the functions $(x, y) \mapsto u_{1}(x) u_{2}(y)$ where for $i=1,2, u_{i}: M_{i} \rightarrow \mathbb{R}$ is an eigenfunction for the eigenvalue $\mu_{i}$ for $\Delta_{g_{i}}$, where $\mu_{1}+\mu_{2}=\lambda_{1}\left(M_{1}, g_{1}\right)$. It then follows from (64) that

$$
K_{0}=\left\{(x, y) \in M \mapsto \varphi(x) / \varphi \in \Lambda_{1}\left(M_{1}, g_{1}\right)\right\},
$$

where

$$
\Lambda_{1}\left(M_{1}, g_{1}\right):=\left\{\varphi \in C^{2}\left(M_{1}\right) / \Delta_{g_{1}} \varphi=\lambda_{1}\left(M_{1}, g_{1}\right) \varphi\right\}
$$

is the eigenspace associated to the first eigenvalue $\lambda_{1}\left(M_{1}, g_{1}\right)$. We claim that

$$
\int_{M_{1}} \varphi^{3} d v_{g_{1}}=0 \text { for all } \varphi \in \Lambda_{1}\left(M_{1}, g_{1}\right) .
$$

We prove the claim. In Case (i), since $d \geq 3$ and $\tilde{u}_{0}$ is a local minimizer, (65) follows from (51) and (54). In Case (ii), since $\left(M_{1}, g_{1}\right)=\left(\mathbb{S}^{d}(r)\right.$, can), $\Lambda_{1}\left(M_{1}, g_{1}\right)$ is the restriction to $\mathbb{S}^{d}(r)$ of linear functions on $\mathbb{R}^{d+1}$, and then (65) follows from symmetry. This proves the claim. 
We claim that $I_{0}^{\prime \prime}\left(u_{0}\right) \geq 0$. We prove the claim. Indeed, since $I_{0}^{\prime}\left(u_{0}\right)=0$, straightforward computations yield

$$
I_{0}^{\prime \prime}\left(u_{0}\right)(v, v)=\frac{2 I_{0}\left(u_{0}\right)}{\left\|u_{0}\right\|_{h}^{2}}\left(\|v\|_{h}^{2}-\left(2^{\star}-1\right) \int_{M} u_{0}^{2^{\star}-2} v^{2} d v_{g}\right)
$$

for all $v \in\left(\mathbb{R} u_{0}\right)^{\perp} \subset H_{1}^{2}(M)$. With the choice of $u_{0}$ and $h$ in (63), we then get that

$$
I_{0}^{\prime \prime}\left(u_{0}\right)(v, v)=\frac{2 I_{0}\left(u_{0}\right)}{\left\|u_{0}\right\|_{h}^{2}}\left(\int_{M}|\nabla v|_{g}^{2} d v_{g}-\lambda_{1}\left(M_{1}, g_{1}\right) \int_{M} v^{2} d v_{g}\right)
$$

for all $v \in\left(\mathbb{R} u_{0}\right)^{\perp} \subset H_{1}^{2}(M)$. Therefore, it follows from the above characterization of $K_{0}$ above that $I_{0}^{\prime \prime}\left(u_{0}\right)(v, v) \geq 0$ for all $v \in\left(\mathbb{R} u_{0}\right)^{\perp}$. Since $u_{0}$ is in the kernel of $I_{0}^{\prime \prime}\left(u_{0}\right)$, we then get that $I_{0}^{\prime \prime}\left(u_{0}\right) \geq 0$. This proves the claim.

Since the elements of $K_{0}$ are independent of the second variable, we get that

$$
\left(\Delta_{g}-\lambda_{1}\left(M_{1}, g_{1}\right)\right)^{-1}\left((x, y) \mapsto \varphi^{2}(x)\right)=(x, y) \mapsto\left(\Delta_{g_{1}}-\lambda_{1}\left(M_{1}, g_{1}\right)\right)^{-1}\left(\varphi^{2}(x)\right)
$$

for all $\varphi \in \Lambda_{1}\left(M_{1}, g_{1}\right)$ where $\left(\Delta_{g_{1}}-\lambda_{1}\left(M_{1}, g_{1}\right)\right)^{-1}$ is the inverse of the isomorphism

$$
\begin{array}{clc}
\Lambda_{1}\left(M_{1}, g_{1}\right)^{\perp} & \rightarrow & \left(\Lambda_{1}\left(M_{1}, g_{1}\right)^{\perp}\right)^{\prime} \\
\phi & \mapsto \quad\left(\tau \mapsto \int_{M_{1}}\left((\nabla \phi, \nabla \tau)_{g_{1}}-\lambda_{1}\left(M_{1}, g_{1}\right) \phi \tau\right) d v_{g_{1}}\right)
\end{array}
$$

where the orthogonality in $H_{1}^{2}\left(M_{1}\right)$ is considered with respect to the standard $L^{2}$-product. As a consequence, the expression (61) can be rewritten

$$
\begin{array}{r}
A_{4}(\varphi)=\frac{c_{1} \operatorname{Vol}_{g_{2}}\left(M_{2}\right)}{2}\left(-\left(2^{\star}-1\right) \lambda_{1}\left(M_{1}, g_{1}\right) \int_{M_{1}} \varphi^{2}\left(\Delta_{g_{1}}-\lambda_{1}\left(M_{1}, g_{1}\right)\right)^{-1}\left(\varphi^{2}\right) d v_{g_{1}}\right. \\
\left.-\frac{2^{\star}-3}{3} \int_{M_{1}} \varphi^{4} d v_{g_{1}}\right)
\end{array}
$$

for all $\varphi \in K_{0}$, where $c_{1}:=\left(2^{\star}-1\right)\left(2^{\star}-2\right) u_{0}^{2^{\star}-4}$ and, for simplicity, we have written $K_{0}$ for $\Lambda_{1}\left(M_{1}, g_{1}\right)$. We now distinguish Cases (i) and (ii) of Proposition 7.3

Case (i): $d \geq 3$ and $\tilde{u}_{0}$ is a local minimizer. As one checks,

$$
\tilde{K}_{0}:=\left\{\varphi \in C^{2}\left(M_{1}\right) / \Delta_{g_{1}} \varphi+\tilde{h} \varphi=\left(2_{d}^{\star}-1\right) \tilde{u}_{0}^{2_{d}^{\star}-2} \varphi\right\}=\Lambda_{1}\left(M_{1}, g_{1}\right) .
$$

We define $\tilde{A}_{4}$ for $\tilde{u}_{0}$ and therefore (61) yields

$$
\begin{aligned}
& \frac{2}{\left(2_{d}^{\star}-1\right)\left(2_{d}^{\star}-2\right) \tilde{u}_{0}^{2_{d}^{\star}-4}} \tilde{A}_{4}(\varphi) \\
& =-\left(2_{d}^{\star}-1\right) \lambda_{1}\left(M_{1}, g_{1}\right) \int_{M_{1}} \varphi^{2}\left(\Delta_{g_{1}}-\lambda_{1}\left(M_{1}, g_{1}\right)\right)^{-1}\left(\varphi^{2}\right) d v_{g_{1}} \\
& -\frac{2_{d}^{\star}-3}{3} \int_{M_{1}} \varphi^{4} d v_{g_{1}}
\end{aligned}
$$

for all $\varphi \in \Lambda_{1}\left(M_{1}, g_{1}\right)$. Plugging this expression into (66) yields

$$
A_{4}(\varphi)=c_{2} \cdot\left(\frac{\left(2^{\star}-1\right) \tilde{A}_{4}(\varphi)}{4\left(2_{d}^{\star}-1\right)^{2}\left(2_{d}^{\star}-2\right) \tilde{u}_{0}^{2_{d}^{\star}-4}}+\frac{(n-d)}{3(n-2)(d+2)} \int_{M_{1}} \varphi^{4} d v_{g_{1}}\right)
$$


for all $\varphi \in \Lambda_{1}\left(M_{1}, g_{1}\right)$, where $c_{2}:=4\left(2^{\star}-1\right)\left(2^{\star}-2\right) u_{0}^{2^{\star}-4} \operatorname{Vol}_{g_{2}}\left(M_{2}\right)$. In particular, if $\tilde{u}_{0}$ is a local minimizer for $\tilde{I}_{0}$, then (54) yields $\tilde{A}_{4} \geq 0$. Therefore, $A_{4}(\varphi)>0$ for all $\varphi \in \Lambda_{1}\left(M_{1}, g_{1}\right) \backslash\{0\}$ since $n-d>0$. This proves Proposition 7.3 in Case (i).

Case (ii): $\left(M_{1}, g_{1}\right)=\left(\mathbb{S}^{d}(r)\right.$, can $)$. The case $d \geq 3$ is covered by Case (i), and only the cases $d=1,2$ remain to be covered. For simplicity, we assume that $r=1$. It follows from Berger-Gauduchon-Mazet 2 that the second positive eigenvalue $\lambda_{2}\left(\mathbb{S}^{d}\right.$, can $)$ is $2(d+1)$ and the eigenfunctions are the restrictions to $\mathbb{S}^{d}$ of secondorder homogeneous harmonic polynomials on $\mathbb{R}^{d+1}$.

We let Eucl be the Euclidean metric on $\mathbb{R}^{d+1}$. We claim that

$$
\left(\Delta_{\text {can }}-\lambda_{1}\right)^{-1}\left(\varphi^{2}\right)=\frac{\varphi^{2}+\frac{\lambda_{2} \Delta_{\text {Eucl }}\left(\varphi^{2}\right)}{2(d+1) \lambda_{1}}}{\lambda_{2}-\lambda_{1}} \text { for all } \varphi \in \Lambda_{1}\left(\mathbb{S}^{d}, \text { can }\right) .
$$

where $\lambda_{1}=d$ and $\lambda_{2}=2(d+1)$. We prove the claim. We fix $\varphi \in \Lambda_{1}\left(\mathbb{S}^{d}\right.$, can). In particular $\varphi^{2}$ is a second-order homogeneous polynomial on $\mathbb{R}^{d+1}$, and $\varphi^{2}+$ $\frac{\Delta_{\text {Eucl }}\left(\varphi^{2}\right)}{2(d+1)}|x|^{2}$ is a harmonic second-order homogenous polynomial, and therefore its restriction to $\mathbb{S}^{d}$ is an eigenfunction for $\lambda_{2}$. Since $\Delta_{\mathrm{Eucl}}\left(\varphi^{2}\right)$ is constant and $|x|^{2}$ is constant on $\mathbb{S}^{d}$, (67) follows from a direct computation. This proves the claim.

We claim that

$$
\int_{\mathbb{S}^{d}} \varphi^{4} d v_{\text {can }}=-\frac{3}{2(d+3)} \Delta_{\text {Eucl }}\left(\varphi^{2}\right) \int_{\mathbb{S}^{d}} \varphi^{2} d v_{\text {can }} \text { for all } \varphi \in \Lambda_{1}\left(\mathbb{S}^{d}, \text { can }\right) .
$$

We prove the claim. Since, up to homothetic transformation, $\varphi$ is a coordinate function, proving (68) is equivalent to proving $\int_{\mathbb{S}^{d}} x^{4} d v_{\mathrm{can}}=(3 /(d+3)) \int_{\mathbb{S}^{d}} x^{2} d v_{\mathrm{can}}$ where $x$ is the first coordinate in $\mathbb{R}^{d+1}$. This latest identity follows from the change of variable $(t, \sigma) \mapsto\left(t, \sqrt{1-t^{2}} \sigma\right)$ from $(-1,1) \times \mathbb{S}^{d-1}$ to $\mathbb{S}^{d} \backslash\{( \pm 1, \ldots, 0)\}$. This proves the claim.

Plugging (67) and (68) into (66) yields

$$
A_{4}(\varphi)=\frac{4\left(2^{\star}-1\right)\left(2^{\star}-2\right) u_{0}^{2^{\star}-4} \operatorname{Vol}_{g_{2}}\left(M_{2}\right)(n-d)}{3(n-2)(d+2)} \int_{\mathbb{S}^{d}} \varphi^{4} d v_{\mathrm{can}}
$$

for all $\varphi \in \Lambda_{1}\left(\mathbb{S}^{d}\right.$, can). In particular, since $d<n$, we have that $A_{4}(\varphi)>0$ for all $\varphi \in \Lambda_{1}\left(\mathbb{S}^{d}\right.$, can $) \backslash\{0\}$. This proves Case (ii) of Proposition 7.3 when $r=1$. The general case follows by rescaling. This proves Proposition 7.3 .

As a remark, the computations made for Case (ii) are valid when $d=n \geq 3$ (that is $M=\mathbb{S}^{d}=\mathbb{S}^{n}$ ), and we get that $A_{4} \equiv 0$, which has been obtained by another method in Proposition 7.2 .

When $h \equiv c_{n} R_{g}$, an immediate consequence of Proposition 7.3 is the following:

Corollary 7.1. Let $\left(N, g_{N}\right)$ be a compact Riemannian manifold of positive constant scalar curvature. We choose $d \geq 1$ and we assume that

$$
R_{g_{N}}<\operatorname{dim}(N) \lambda_{1}\left(N, g_{N}\right) \text { and } n:=d+\operatorname{dim}(N) \geq 3 .
$$

We endow the manifold $M:=\mathbb{S}^{d}\left(\sqrt{\operatorname{dim}(N) \cdot d / R_{g_{N}}}\right) \times N$ with the product metric $g:=\operatorname{can} \oplus g_{N}$. Then the positive constant solution to the scalar curvature equation $\Delta_{g} u+c_{n} R_{g} u=u^{2^{\star}-1}$ on $M$ is a degenerate strict local minimizer. 
Proof of Corollary 7.1. We fix $r_{0}:=\sqrt{\operatorname{dim}(N) \cdot d / R_{g_{N}}}$. It follows from inequality (69) that we are in Case (ii) of Proposition 7.3. With this choice of $r_{0}$, we have that

$$
c_{n} R_{g}=\frac{n-2}{4(n-1)}\left(R_{g_{N}}+\frac{d(d-1)}{r_{0}^{2}}\right)=\frac{(n-2) d}{4 r_{0}^{2}}=\frac{\lambda_{1}\left(\mathbb{S}^{d}\left(r_{0}\right), \mathrm{can}\right)}{2^{\star}-2} .
$$

Therefore Proposition 7.3 applies. This proves Corollary 7.1 .

Inequality (69) holds if $g_{N}$ is a Yamabe metric, that is a minimizer of the Yamabe functional. From the pde point of view, a metric $g$ on $M$ is a Yamabe metric iff $R_{g}$ is constant and the minimum of $I_{0}$ (with $h \equiv c_{n} R_{g}$ ) is achieved by constants.

As a remark, Corollary 7.1 can be generalized by replacing the sphere by a manifold $V$ of dimension $d \geq 3$ with a degenerate Yamabe metric $g_{V}$ of positive scalar curvature satisfying $R_{g_{N}}=\operatorname{dim}(N) \lambda_{1}\left(V, g_{V}\right)$ and $\lambda_{1}\left(V, g_{V}\right)<\lambda_{1}\left(N, g_{N}\right)$. Note that the degeneracy of $g_{V}$ implies that $R_{g_{V}}=(\operatorname{dim}(V)-1) \lambda_{1}\left(V, g_{V}\right)$.

\section{REFERENCES}

[1] A. Ambrosetti and A. Malchiodi, Perturbation methods and semilinear elliptic problems on $\mathbf{R}^{n}$, Progress in Mathematics, vol. 240, Birkhäuser Verlag, Basel, 2006.

[2] M. Berger, P. Gauduchon, and E. Mazet, Le spectre d'une variété riemannienne, Lecture Notes in Mathematics, Vol. 194, Springer-Verlag, Berlin, 1971.

[3] G. Bianchi and H. Egnell, A note on Sobolev inequality, J. Funct. Anal. 100 (1991), 18-24.

[4] S. Brendle and F. C. Marques, Blow-up phenomena for the Yamabe equation. II, J. Differential Geom. 81 (2009), no. 2, 225-250.

[5] J. Byeon and L. Jeanjean, Standing waves for nonlinear Schrodinger equations with a general nonlinearity, Arch. Rational Mech. Anal. 185 (2007), no. 2, 185-200.

[6] W. Chen, J. Wei, and S. Yan, Infinitely many solutions for the Schrödinger equations in $\mathbb{R}^{N}$ with critical growth, J. Differential Equations 252 (2012), no. 3, 2425-2447.

[7] E. N. Dancer, Real analyticity and non-degeneracy, Math. Ann. 325 (2003), no. 2, 369-392.

[8] _ Peak solutions without non-degeneracy conditions, J. Differential Equations 246 (2009), no. 8, 3077-3088.

[9] M. del Pino and P.L. Felmer, Local mountain passes for semilinear elliptic problems in unbounded domains, Calc. Var. Partial Differential Equations 4 (1996), no. 2, 121-137.

[10] M. del Pino, M. Musso, F. Pacard, and A. Pistoia, Large energy entire solutions for the Yamabe equation, J. Differential Equations 251 (2011), no. 9, 2568-2597.

[11] - Torus action on $S^{n}$ and sign-changing solutions for conformally invariant equations, Ann. Sc. Norm. Super. Pisa Cl. Sci. (5) 12 (2013), no. 1, 209-237.

[12] O. Druet, Compactness for Yamabe metrics in low dimensions, Int. Math. Res. Not. 23 (2004), 1143-1191.

[13] O. Druet and E. Hebey, Blow-up examples for second order elliptic PDEs of critical Sobolev growth, Trans. Amer. Math. Soc. 357 (2005), no. 5, 1915-1929 (electronic).

[14] P. Esposito, A. Pistoia, and J. Vétois, The effect of linear perturbations on the Yamabe problem, Math. Ann. 358 (2014), no. 1-2, 511-560.

[15] E. Hebey and J. Wei, Resonant states for the static Klein-Gordon-Maxwell-Proca system, Math. Res. Lett. 19 (2012), no. 4, 953-967.

[16] L. Jeanjean and K. Tanaka, Singularly perturbed elliptic problems with superlinear or asymptotically linear nonlinearities, Calc. Var. Partial Differential Equations 21 (2004), 287-318.

[17] M. A. Khuri, F. C. Marques, and R. M. Schoen, A compactness theorem for the Yamabe problem, J. Differential Geom. 81 (2009), no. 1, 143-196.

[18] O. Kobayashi, Yamabe metrics and conformal transformations, Tohoku Math. J. (2) 44 (1992), no. 2, 251-258.

[19] J.M. Lee and T.H. Parker, The Yamabe problem, Bull. Amer. Math. Soc. 17 (1987), 37-91.

[20] M. Musso and J. Wei, Nondegeneracy of nonradial nodal solutions to Yamabe problem. Preprint. 
[21] M. Obata, The conjectures on conformal transformations of Riemannian manifolds, J. Differential Geom. 6 (1971/72), 247-258.

[22] A. Pistoia and J. Vétois, Sign-changing bubble towers for asymptotically critical elliptic equations on Riemannian manifolds, J. Differential Equations 254 (2013), no. 11, 4245-4278.

[23] O. Rey, The role of the Green's function in a nonlinear elliptic equation involving the critical Sobolev exponent, J. Funct. Anal. 89 (1990), no. 1, 1-52.

[24] F. Robert and J. Vétois, Sign-Changing Blow-Up for Scalar Curvature Type Equations, Comm. Part. Differ. Equat. 38 (2013), no. 8, 1437-1465.

[25] _ A general theorem for the construction of blowing-up solutions to some elliptic nonlinear equations via Lyapunov-Schmidt's reduction, Concentration Compactness and Profile Decomposition (Bangalore, 2011), Trends in Mathematics, Springer, Basel, 2013, pp. 85-116.

[26] R.M. Schoen, On the number of constant scalar curvature metrics in a conformal class, Differential geometry, Pitman Monogr. Surveys Pure Appl. Math., vol. 52, Longman Sci. Tech., Harlow, 1991, pp. 311-320.

Frédéric Robert, Institut Élie Cartan, UMr 7502, Université de Lorraine, BP 70239, F-54506 Vandeuvre-Lès-Nancy, France; Pacific Institute for the Mathematical Sciences, UMi CNRS 3069, 4176-2207 Main Mall, Vancouver, BC, V6T 1Z4, Canada

E-mail address: frederic.robert@univ-lorraine.fr

Jérôme Vétois, Université de Nice Sophia Antipolis, Laboratoire J.-A. Dieudonné, CNRS UMR 7351, Parc Valrose, F-06108 Nice Cedex 2, France

E-mail address: vetois@unice.fr 